\title{
Centric Allometry: Studying Growth Using Landmark Data
}

\author{
Fred L. Bookstein ${ }^{1,2} \mathbb{0}$
}

Received: 29 July 2020 / Accepted: 26 December 2020 / Published online: 2 February 2021

(c) The Author(s) 2021

\begin{abstract}
The geometric morphometric (GMM) construction of Procrustes shape coordinates from a data set of homologous landmark configurations puts exact algebraic constraints on position, orientation, and geometric scale. While position as digitized is not ordinarily a biologically meaningful quantity, and orientation is relevant mainly when some organismal function interacts with a Cartesian positional gradient such as horizontality, size per se is a crucially important biometric concept, especially in contexts like growth, biomechanics, or bioenergetics. "Normalizing" or "standardizing" size (usually by dividing the square root of the summed squared distances from the centroid out of all the Cartesian coordinates specimen by specimen), while associated with the elegant symmetries of the Mardia-Dryden distribution in shape space, nevertheless can substantially impeach the validity of any organismal inferences that ensue. This paper adapts two variants of standard morphometric leastsquares, principal components and uniform strains, to circumvent size standardization while still accommodating an analytic toolkit for studies of differential growth that supports landmark-by-landmark graphics and thin-plate splines. Standardization of position and orientation but not size yields the coordinates Franz Boas first discussed in 1905. In studies of growth, a first principal component of these coordinates often appears to involve most landmarks shifting almost directly away from their centroid, hence the proposed model's name, "centric allometry." There is also a joint standardization of shear and dilation resulting in a variant of standard GMM's "nonaffine shape coordinates" where scale information is subsumed in the affine term. Studies of growth allometry should go better in the Boas system than in the Procrustes shape space that is the current conventional workbench for GMM analyses. I demonstrate two examples of this revised approach (one developmental, one phylogenetic) that retrieve all the findings of a conventional shape-space-based approach while focusing much more closely on the phenomenon of allometric growth per se. A three-part Appendix provides an overview of the algebra, highlighting both similarities to the Procrustes approach and contrasts with it.
\end{abstract}

Keywords Centric allometry · Allometric growth · Procrustes analysis · Centroid Size $\cdot$ Uniform component · Shape coordinates $\cdot$ Boas coordinates $\cdot$ Landmark data

\section{Introduction: A Suggestive "new" Diagram Style from 1905}

Many students of geometric morphometrics (GMM) as applied in evolutionary biology will already have encountered some version of Fig. 1a, the standard display of the Procrustes shape coordinates for the neuroanatomical octagon of midsagittal landmarks from the Vilmann data set of

Fred L. Bookstein

flb@stat.washington.edu

1 Department of Evolutionary Biology, University of Vienna, Vienna, Austria

2 Department of Statistics, University of Washington, Seattle, USA laboratory rodents radiographed longitudinally at eight ages (7 days, 14, 21, 30, 40, 60, 90, and 150 days). The raw data, initially published as Appendix A.4.5 of (Bookstein 1991), are currently among the archives of several on-line GMM data resources. My analyses in this paper are restricted to the 18 animals (out of an original 21) with complete data for all eight landmarks at all eight ages. By calling these coordinates "Procrustes shape coordinates" one means that they have been standardized specimen by specimen in four different aspects: horizontal average coordinate, vertical average coordinate, orientation (zero net rotation per specimen around the average, see Appendix A.2), and squared Centroid Size (CS), which is the summed squared distances of all the landmarks from their centroid case by case. 


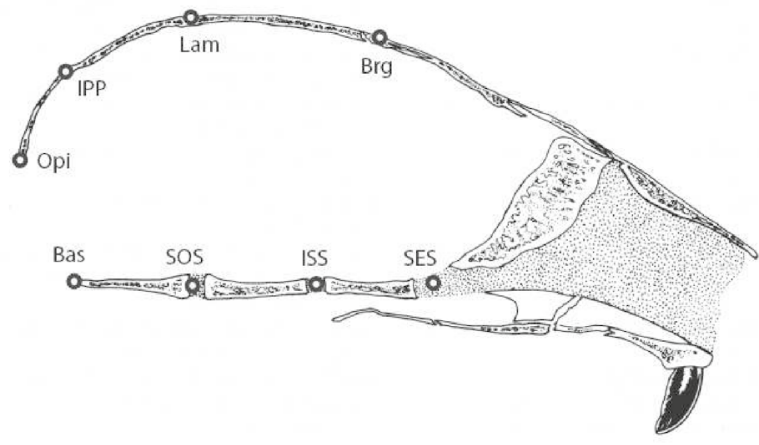

(A) Vilmann data,

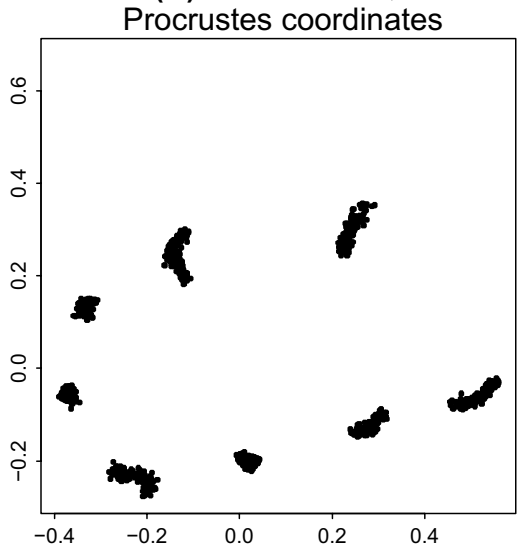

Fig. 1 Aspects of the standard Procrustes analysis for the Vilmann data set. a Scatter of all eight Procrustes shape coordinate pairs for 18 animals radiographed at eight ages each. b Thin-plate spline (deformation) visualization of a convenient multiple of the first relative warp of these data with respect to the overall average shape. c Superposition, still in Procrustes pose, of the average shapes of the animals

This analysis, which first appeared in Bookstein (1991), has been reproduced in many later publications, including several of the standard textbooks (Dryden and Mardia 1998, 2016; Bookstein 2014, 2018), and the data set it visualizes has served as exemplar for diverse advanced GMM analyses, such as integration (Bookstein 2015), factor analysis (Bookstein 2017), and a variant of descriptive finite-element analysis (Bookstein 2018: Sect. 5.2) borrowed from biomechanics. ${ }^{1}$ For instance, Fig. $1 \mathrm{~b}$ shows the first relative warp (RW, shape principal component) of these 144 configurations in the usual iconographic, a thin-plate spline. This particular grid represents the deformation from the sample

\footnotetext{
${ }^{1}$ Indeed, these Vilmann data probably constitute the single most intensively re-analyzed and re-re-analyzed data set to be found anywhere in morphometrics. That renders the perspicuity of its role in introducing the new model (centric allometry) here all the more unexpected.
}

(C) Proc. means, ages 7 and 150 days

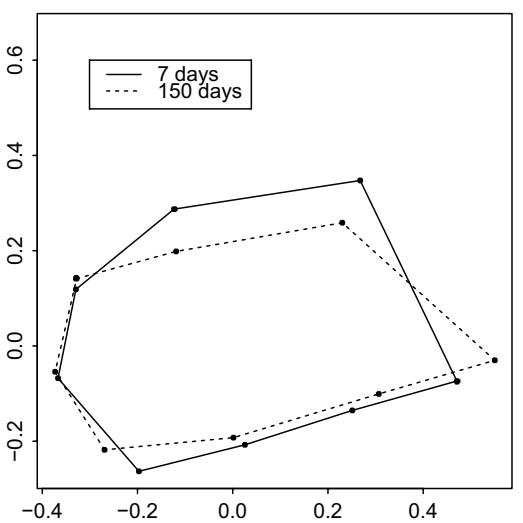

at age 7 (solid lines) and age 150 (dashed lines). Illustration at top: anatomy of the eight landmarks in situ, in anatomical orientation instead of the Procrustes orientation of the other panels. Clockwise from left: Opisthion, Interparietal point, Lambda, Bregma, Sphenoethmoid synchondrosis, Intersphenoidal synchondrosis, Sphenoöccipital synchondrosis, Basion average shape to its modification by a suitable multiple of this relative warp (here, four standard deviations of the corresponding principal component score) in the direction of greater age. This first RW is indeed strongly correlated with age. This is already clear in the display of Fig. 1c, the average shapes at the two extremes of age: clearly cranial base length changes with age by a greater factor than height above it does among the features that leap to the eye.

Since RW1 is correlated with age and age in turn is correlated with size, it might be useful to display the actual effect of age on form, not just on shape. To do so, it is most straightforward to "put size back into" the analysis using the same size measure CS that was used to standardize the size of all the specimens during the Procrustes normalization. This is arranged not by augmenting the shape coordinates by one additional scalar for log Centroid Size (the prescription for rising from Procrustes shape space to form space, see Mitteroecker et al. 2004; Bookstein 2018) but instead by multiplying each configuration of Procrustes 


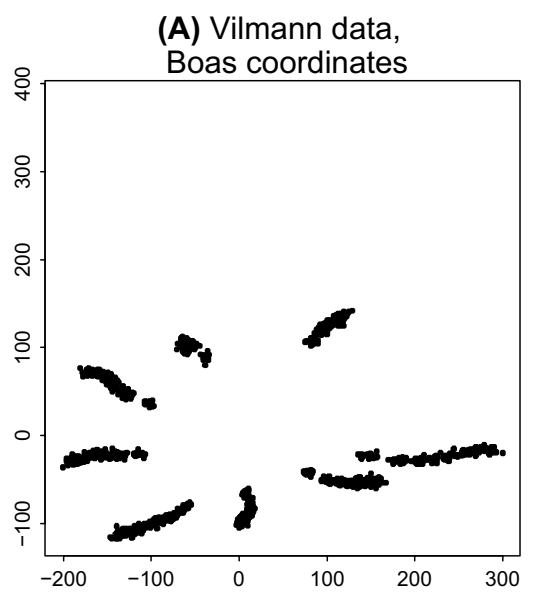

Fig. 2 Analogue of Fig. 1 for the Boas coordinates that back out the scaling step of the Procrustes algorithm. Orientation remains the Procrustes orientation (principal axes horizontal and vertical) already used in Fig. 1. a Centered, reoriented coordinates to the original scale as digitized by Melvin Moss around 1980. b First principal component of these 16 Cartesian coordinates, visualized by a thin-

shape coordinates by the same factor of CS that was divided out in the course of producing those Procrustes shape coordinates in the first place. In this paper these remultiplied configurations will be called Boas coordinates, as in Bookstein (2018), for reasons to be explained in the next section. To restore the functional for size that was divided out is almost equivalent to omitting one of the four standardizations in the standard generalized Procrustes analysis (GPA), namely, the scaling of each form to unit Centroid Size. (Its omission alters the average form, but only very, very slightly: see Appendix A.2.) The horizontal and vertical centering and the rotation to zero net rotation specimen by specimen around the common centroid remain.

After this modification (a truncation, really) of the standard Procrustes algorithm, the scatter of the 144 octagonal configurations appears as the remarkably different-looking display in Fig. 2a. Figure $2 b$ shows the thin-plate spline for the first principal component of the coordinates in Fig. 2a using the same convention as in Fig. 1b, deformation from the mean to a suitable multiple of this component, while Fig. 2c compares the average over the 18 age- 7 configurations to their average over the 18 age- 150 configurations, for ease in appreciating spatial aspects of the growth rates of various interlandmark distances (see Bookstein 2018, Fig. 5.21).

Figures $1 \mathrm{~b}$ and $2 \mathrm{~b}$ are almost identical. In other words, the statistical analysis of the growth of this rodent neuroanatomical octagon proceeds just as well using the Boas coordinates that canceled the Procrustes scaling step as it does using the standard Procrustes procedure with scaling. Better, in fact: from Fig. $2 c$ one can extract the actual growth
(C) Boas means, ages 7 and 150 days

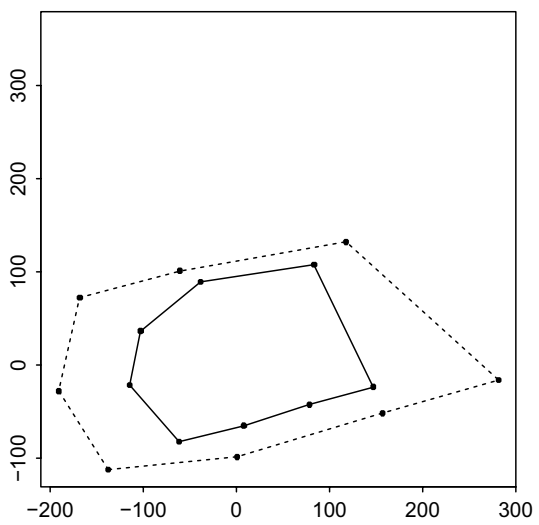

plate spline of the average form. c Averages of the octagons for the age-7 animals (solid lines) and age-150 animals (dashed lines). While frames $\mathbf{b}$ are virtually identical between this figure and the preceding figure, frames a and $\mathbf{c}$ are entirely different as the scaling step of the Procrustes algorithm is accepted (Fig. 1) or backed out (Fig. 2)

rates along diverse transects of these octagons, calibrations that are inaccessible from the Procrustes analogue in Fig. 1c.

But other aspects of the multivariate analysis have changed drastically. First, its language: the algebra of Fig. 1 is least-squares in "Procrustes distance," which has no physical units, whereas that for Fig. 2 is least-squares in ordinary Euclidean distance on the digitizing tablet or the printed page, so that the variance explained by this and subsequent decompositions of the Boas coordinates comes in units of ordinary squared Euclidean distance, perhaps $\mathrm{mm}^{2}$. Partly as a consequence of this transformation of metric structure in the context of the actual process, growth allometry, that this data set is illustrating, the scatter of the first two relative warp scores in Boas space, Fig. 3b, is enormously different from that of the corresponding pair for the usual Procrustes coordinates, Fig. 3a. Principal component 2 (PC2) of the Boas coordinates is far weaker than relative warp 2 (RW2) of the Procrustes shape coordinates, for instance, while the separation of the age- 7 forms from the others (lower left corner) is much more pronounced in the Boas setting, as is the excessive size of the single largest form in the data set (panel B, solid dot at PC1 $=122, \mathrm{PC} 2=1$ ). The formulas for the horizontal coordinate in these two schemes have been graphed as thin-plate splines in Figs. $1 \mathrm{~b}$ and $2 \mathrm{~b}$; the vertical coordinates will be diagrammed presently.

The most important contrast between the analyses is the discrepancy between the two graphical versions of the formula for the first principal component that, as we have seen, represents growth by equivalent diagrams of gradients in both of the analyses. Figure 4 a shows RW1 as a displacement of each average shape coordinate by a vector corresponding to that RW's loadings at length 0.5 (in 


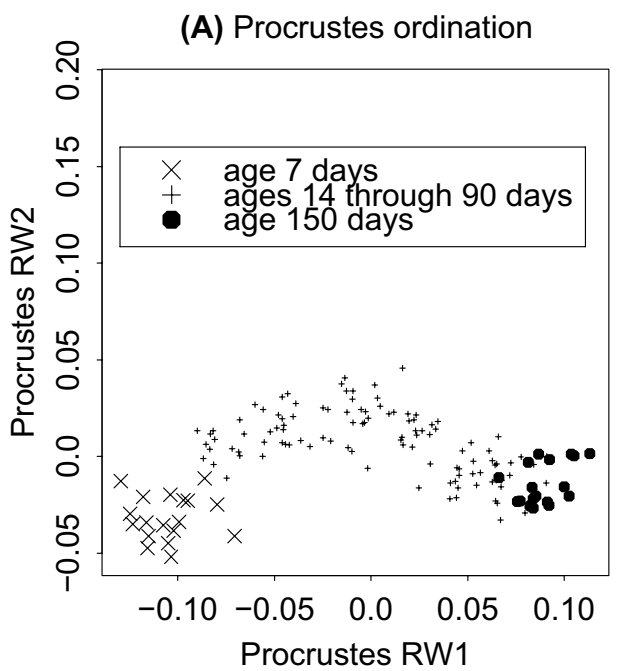

Fig. 3 Scatters of the first two principal component (PC) scores for these 144 landmark configurations. These dimensions are also called the principal coordinates of the squared Procrustes distances and Boas distances, respectively (summaries of the sums of squared coordinate differences from specimen to specimen in Fig. 1a or 2a). In both frames, the youngest animals are at the left. a For the Procrustes shape coordinates. Horizontal, relative warp 1 (RW1); vertical, RW2.
(B) Boas ordination

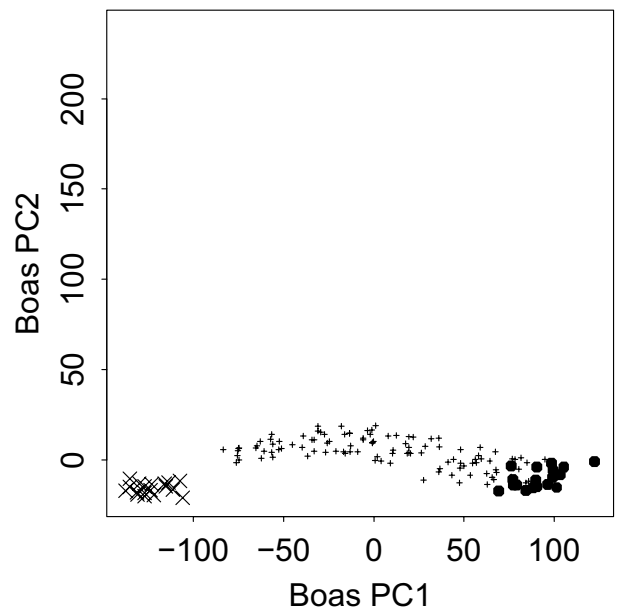

b For the Boas coordinates, PC1 likewise horizontal. In both panels the ordinate is uncorrelated with age, but it represents a far lower proportion of variance in panel $\mathbf{b}$, the Boas analysis. Also note the clarity of the separation between the youngest 18 configurations in panel $\mathbf{b}$ vis-á-vis that in a. Symbols: $\times$, age- 7 animals; •, age-150 animals; + , animals at the other six ages (14 days, $21,30,40,60$, and 90 days)
Fig. 4 Two representations of centric allometry, the diagrammatic innovation put forward in this essay. a As applied to the Procrustes mean shape, scaled to the mean at the oldest age, Fig. 1c. b As applied to the Boas mean form, again scaled to to the mean at the oldest age, Fig. 2c. The text argues that the representation in panel $\mathbf{b}$ is superior by far as a representation of growth allometry, the real biological process that this data set was explicitly designed to capture. BPC1: First principal component of these Boas coordinates
(A) Proc RW1 versus mean shape

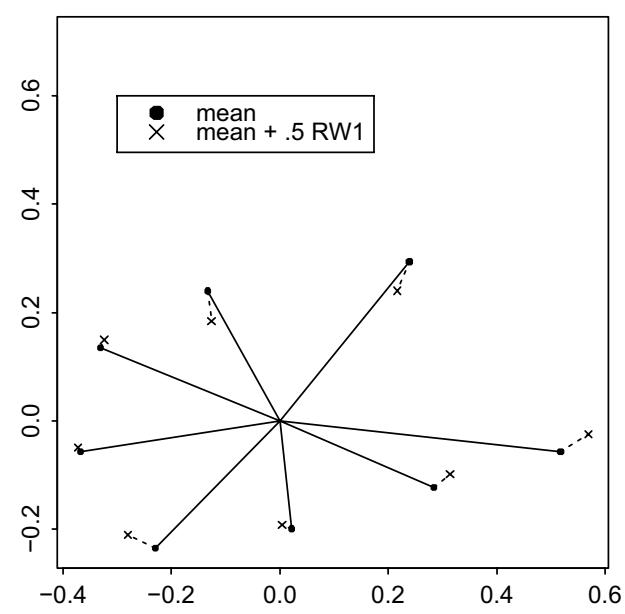

(B) Boas PC1 versus mean form
$($ same scale)

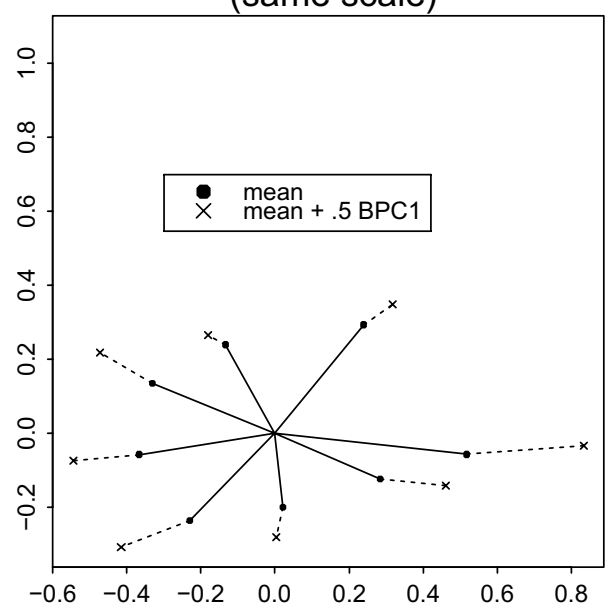

units of sums of squared Procrustes distances accounted for); Fig. 4b, the same for the loadings of Boas PC1 as displacements from each average Boas coordinate, in units of squared Euclidean distance accounted for. The Boas display is much more suggestively structured than the Procrustes display. Figure $4 \mathrm{~b}$ summarizes the Vilmann growth pattern as a centric allometry, displacement of all the landmarks nearly radially outward from the centroid, albeit at rates that vary from landmark to landmark. The Procrustes display, in spite of being a representation of the same growth process, permits no such simple verbal summary-some landmarks appear displaced outward along a central radius, others inward, and still others are displaced oblique to their radii at up to $90^{\circ} .^{2}$

\footnotetext{
2 Vilmann's own studies of this data set (cf. Moss et al. 1983, 1984) began in 1968, prior to Gower's (1975) first announcement of the generalized Procrustes method on which both the standard toolkit of geometric morphometrics and the Boas analysis of this paper are built. Like every other craniofacial biologist back then, Vilmann seems to have been unaware of that novel analysis as a biometric possibility. (Note in this connection that the Gower paper appeared in a journal of psychology, not biology.)
} 
Fig. 5 Demonstration of the equivalence of Procrustes RW1 and Boas PC1 as deformations. a Contrast of the two octets of displacement vectors, exaggerated threefold, after BPC1 is projected down into Procrustes shape space. b The same after RW1 is scaled up into Boas space, this time without exaggeration of their contrast. Open circles: shifts corresponding to RW1. Solid circles: shifts corresponding to $\mathrm{BPC} 1$. Asterisks: landmark-by-landmark means in the appropriate space, weighted by the inverse of Centroid Size in $\mathbf{a}$ and unweighted in $\mathbf{b}$
(A) in Procrustes space

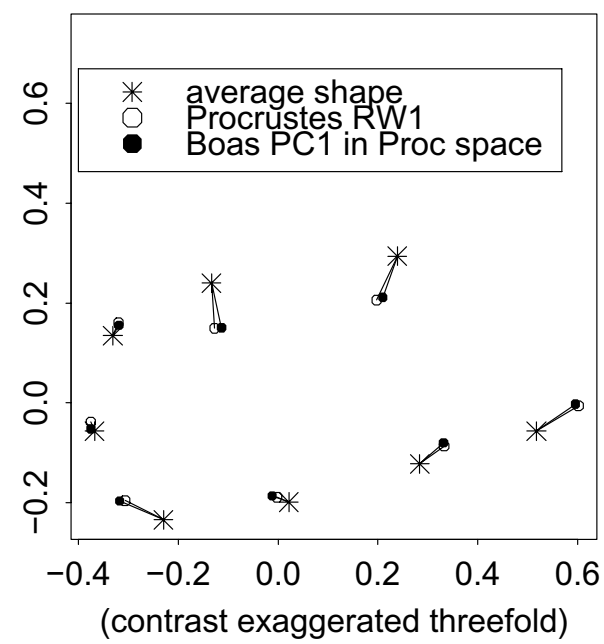

(B) in Boas space

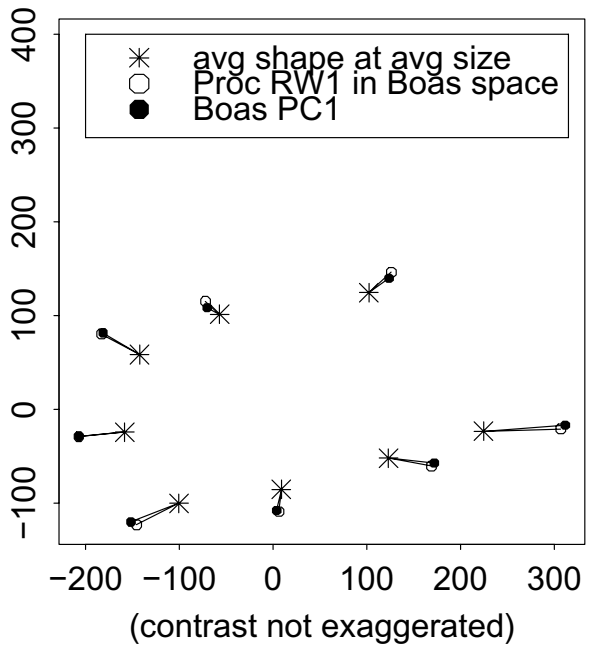

But notice that the shape of the configuration of $\times$ symbols is the same between the panels of Fig. 4-it is more or less the shape of the age-150 average configuration. In effect, all that has changed between the panels is the scaling of that imputed mean configuration with respect to the final average form. Yet only in Fig. 4b, the Boas representation of the data in their original Cartesian units, does the graphic rendition of $\mathrm{PC} 1$ align with the correct biological explanation (allometric growth) of this difference between the octagons. Insofar as rotation is not a component of the allometric style of explanation, it is Fig. 4b, not Fig. 4a, that authorizes the investigator to omit any remark beyond the apparent irrelevance of such a consideration.

The caption of Fig. 2 claims that Figs. $1 \mathrm{~b}$ and $2 \mathrm{~b}$ are "virtually identical," and indeed they do look quite similar. Yet the two panels of Fig. 3 do not much resemble each other, nor the two panels of Fig. 4. A claim that the methods are numerically equivalent - that the deformations of RW1 and BPC1 are almost exactly the same when interpreted as transformation grids-must be based on more than a subjective judgment. Figure 5 presents the necessary argument in explicit graphical form. The two panels here correspond not to the two types of analysis (that antinomy is coded in the plotting symbols instead) but to the two different spaces, Procrustes space or Boas space, in which the demand for a quantitative confrontation might be couched. In panel A the pair of linear composite scores are plotted by their coefficients as they would be diagrammed in Procrustes space. The open circles are the coefficients of the first conventional relative warp of these Procrustes shape coordinate octagons, plotted in correct proportion as vectors out of the Procrustes average shape. The solid circles are a different quantification, a sort of hybrid whereby the displacements of Boas coordinates in their Cartesian space are Procrustes-fitted to the original mean form by an adjustment of their size (an adjustment that is, of course, nonsense in the Boas context per se). When the two composites, RW1 and BPC1, are plotted to the same net Procrustes magnitude, the octets of vectors would have plotted directly atop each other had I not exaggerated the contrast between them by a factor of three before plotting. The crossproduct of these two 16-vectors is 0.99956 , corresponding to an angle in their space of about $1.7^{\circ}$ (the angle that the minute hand of a clock rotates in about $17 \mathrm{~s})$.

In panel B the same hybrid display is presented in Boas space instead of Procrustes space. The solid circles are now just the displacements corresponding to Boas PC1, whereas the open circles correspond to landmark-by-landmark displacements of Procrustes RW1 after a rescaling (an adjustment that is, of course, nonsense in the Procrustes context) in order to have the same ratio of net Euclidean magnitudes (Centroid Sizes). Here the contrast is marginally visible without any need for the threefold magnification. From either panel one draws two obvious conclusions: first, the deformations we are discussing - the deformations of allometric growth of these rat neurocranial octagons-agree as regards their gradients everywhere across the form; but, second, the interpretation of those gradients in terms of Cartesian coordinates is entirely different between the two spaces.

In view of this identity of meaning between the two panels of Fig. 4 (up to a scaling step that can easily be shown to be biologically meaningless, see Appendix A.1), the contrast between the two panels of Fig. 3 becomes even more salient, inasmuch as the horizontal coordinates of these two plots are so obviously not the same between the analyses. That is, of course, because Centroid Size, a specific correlate of the allometric growth under study, has been divided out of the analysis in panel A but remains implicit in panel B. The inescapable conclusion is that the score on Procrustes $R W 1$ is irrelevant to the understanding of allometric growth, 
precisely because it does not capture the appropriate sizedependence explicitly modeled in BPC1.

The advantage of the Boas analysis in a study of growth allometry is particularly evident in one specific aspect of Fig. 4b, the near-collinearity between each solid segment and its matching dashed segment. Such an alignment permits a description in which each landmark grows away from their common center without much additional rotation, so that the distances from the center at these fixed angles become a sufficient summary of the growth pattern. Moreover, the ratio of the two distances at each landmark is a good representation of the specific growth rate assignable to that landmark: maximal at the two ends of the cranial base, minimal at the landmarks in-between and along the top of the calva. The Procrustes diagram, Fig. 4a, proffers no such description in millimeters, as every verbalization of the pattern there would have to include the word "relative"- the relative rate of expansion of height with respect to increase of cranial base length, etc. Whenever the approximate collinearity so apparent in Fig. 4b obtains, the first Boas PC, by remaining in units of length, is better serving the function that Centroid Size was designed to serve in the Procrustes approach: a "best possible" size measure. In other words, when BPC1 manifests this collinear pattern, the diagram assures us that those aspects of form being ignored by the centroid-to-landmark distance description - the rotations of the individual landmarks around the pooled version that kept the whole configuration rigid - are negligible with respect to the growth changes at $90^{\circ}$ to them, the growth rates of these centric distances themselves. Hence the name I have suggested for this technique, centric allometry.

A comparison of Fig. 4b with Figs. 1b or $2 b$ reinforces this "irrotational" property of Boas $\mathrm{PC} 1$ analysis. In either thin-plate spline figure there is a clear contribution to RW1 that is a relative repositioning of the landmark Opi with respect to its pair of neighbors Bas and IPP: the distance Opi-Bas grows much more slowly than the distance Opi-IPP. This effect, however, is azimuthal, not radial: it pertains to distances along the perimeter of this octagon, not outwards from its center. By design, the contrasting style of diagramming a Boas analysis in Fig. $4 \mathrm{~b}$ attenuates this feature-when what is shown is a much smaller multiple of this same first PC, there is only a small hint of an azimuthal effect at Bas. Analyses of this growth data set published earlier have identified this phenomenon in the vicinity of Opi either as an expression of the feature of smallest scale in this octagon, partial warp 5 of the standard toolkit (Bookstein 2018), or as a similarly scale-inappropriate factor that emerges from the alternative factor-analysis machinery of Bookstein (2017). It is indeed part of a description of growth, but it does not participate in the large-scale summary that is our concern in this paper. Put another way, the information that is de-emphasized in Fig. $4 \mathrm{~b}$ about this strong local effect is still present in the display of Fig. 2b, which is a different graphical representation of the same principal component BPC1. Evidently a competent analysis of this data set requires inspection of both.

The question immediately arises of why Fig. 1 was ever constructed - why a study of growth allometry should have been situated in Procrustes shape space at all. Why should this author and every later investigator into the multivariate structure of this classic data set ever have bothered to divide out Centroid Size in investigations where the ultimate organismal interpretation will require that division to be reversed? ${ }^{3}$ For studies along these lines, this paper has introduced a new analysis of landmark configurations that does, indeed, cancel out the effects of this specific biologically inappropriate step in the standard algorithm. Yes, there is a loss of mathematical elegance, several aspects of which will be touched on in the next section; but the gain in empirical explanatory power for the original scientific purpose, that of understanding patterns of organismal growth, more than compensates. Moreover, the standard reduction of form-variation to size, affine shape, and partial warps (see Bookstein 2018, Chapter 5) proceeds in parallel except that size is now folded into the affine component, where it could have been encoded all along.

The outline of the rest of this paper is as follows. Section 2 briefly reviews the reason that Boas coordinates have that name, compiles some early references to the comparative biometrics of organisms in terms of "size" versus "shape," and surveys the small biometric literature that adumbrated this notion of centric allometry a few decades ago from a morphometrically less sophisticated point of view. Section 3 extends the analysis to a maneuver inaccessible from the standard Procrustes toolkit: the joint subsetting of landmark lists along with sample subgroups in order to enhance the power of the hypothesis of growth allometry, as explicated via a simplistic phylogenetic example from hominoid evolution. A closing discussion (Sect. 4) explains why D'Arcy Thompson ought not to be credited with any of these ideas as either evolutionary biologists or developmental biologists now apply them; then considers the competing versions of the role of explicit experimental data in studies of growth as promulgated by Hans Przibram in the 1920's, versus Peter Sneath in the 1960's; and, finally, explores the comparative costs and benefits between scaled and unscaled approaches (analysis of Procrustes coordinates versus Boas coordinates) in the design of biometric tools for analysis of organismal growth. Following these remarks is an Appendix reviewing three aspects of the algebra of the proposed

\footnotetext{
${ }^{3}$ Recall the Russian folktale that ends with Igor asking Ivan, "So, why did we eat those frogs?" See Alan Plater, Oliver's Travels, Little, Brown and Co., 1994.
} 
method-Centroid Size, specimen-by-specimen rotation, and the advantages of the Boas principal component method for representing centric allometry-in greater detail.

\section{GMM and Allometry}

A hundred fifteen years ago the pioneering American anthropologist Franz Boas published a suggestion so simple that it occupied only two columns in the issue of Science in which it appeared (Boas 1905): that superposition of multiple forms in the service of anthropological investigations should not be by any two-point registration (a preferred point and a preferred interlandmark segment out of it, both fixed in position on the page) but instead by a simple criterion of least squares. ${ }^{4}$ Boas's note is entitled "The horizontal plane of the skull and the general problem of the comparison of variable forms," but its rhetoric treats those two phrases as contrasting, not complementary: his closing comment is that if his new least-squares method is embraced, the "arbitrary element in composite drawings or photographs," namely, the choice of a reference segment between some pair of landmarks to serve as the operationalization of horizontal, "may be eliminated." (This technical issue in craniofacial biology endured for decades: see, for instance, the critique in Moyers and Bookstein (1979).)

To state that the criterion of fit is "least-squares" is in fact to solve the problem in an instant. Boas's algorithm for this fit has two steps: superposition of the centroids of all the forms at a common point, which may as well be the origin of coordinates, and then reorientation around that common centroid to least-squared differences by precisely the same formula that all of our Procrustes toolkits find themselves using here in 2020 - it sets to zero the landmark-weighted reorientation of each form around their average as referred to this common centroid. This is itself an elementary theorem: the least-squares fits between two configurations involve the same rotation whether or not they are rescaled. It follows directly, for instance, from the regression formula (5.7a) on p. 411 of Bookstein (2018), or equivalently from the singular-value notation in Rohlf and Slice (1990). (See Appendix A.2 of this article for details.) Boas's own student Eleanor Phelps, for example, avoided any discussion of shape when she adapted his 1905 method of unscaled superposition to the problem of quantifying variability of different midsagittal landmark configurations (Phelps 1932). All of her

\footnotetext{
${ }^{4}$ It was Theodore Cole, University of Missouri, who, in the bibliographical note Cole (1996), startled the GMM community by calling our attention to Boas's (1905) contribution, the irony of which is central to the intellectual history of all ensuing Procrustes aspects of morphometrics up to and including this article itself. This footnote serves as a belated thank-you for his discovery of Boas's priority.
}

diagrams are explicitly declared to be in centimeters; the technology is unmistakably the same appeal to least-squares as in Boas's original note of 27 years earlier.

Some more recent representations of the variability of cranial form, though not emulating Boas's exploitation of least-squares methodology, have likewise presented unrescaled superpositions in this way: see, for instance, Delattre and Fenart (1960) or Moss et al. 1983. Robert Corruccini's method of Cartesian coordinate analysis (Corruccini 1981) reviews a range of late 20th-century approaches to direct analysis of coordinates, some "following the data normalization procedure advocated by Sneath" (in effect, the Centroid Size scaling of classic Procrustes) and some not. Corruccini's own preference (p. 34) is for the version that we would now call the explicit scaling to Centroid Size unity - the presentation in Fig. 1.

Boas's idea of a superposition that optimizes summed squared specimen-to-specimen landmark distances over position and orientation implicitly assumes that neither position nor orientation is actually a meaningful biological quantity. Both of these propositions are arguable in particular settings. Position surely matters for discussions of functions like locomotion for which lever arms over a landscape are relevant. And orientation matters in at least two different contexts: when function has to do with the local horizontal, or when one aspect of the form is an axis or plane of symmetry. (Boas himself already mentions this latter concern, as in the presence of bilateral symmetry the generalized three-dimensional rotation of a solid skull reduces to a two-dimensional problem.) Speculations on the relevance of criteria like these should be a part of any investigation of landmark coordinate data prior to an invocation of either Boas's algebra or Procrustes algebra in the course of their preparation for some multivariate statistical analysis.

But the discussion must become a great deal more sophisticated when the issue is whether or not to carry out a size standardization. Size per se is a quantification of enormous biological significance for both growth and function, serving as it does variously for biomass, for age, and for aspects of organismal scale that often need to be accommodated in the pursuit of subtler explanations. This multiplicity of roles for size in biomathematics aligns with a distinction of purposes between taxonomy and growth analysis together with the historical accident of an early but widely cited and appreciated application of grids in numerical taxonomy. As Sokal and Sneath (1963, p. 80) put it, "allometry is a problem related to the effect of environment on characters ... and to the problem of redundancy and empirical correlation (that is, the crude measures may depend on a small number of underlying causes)." That redundancy, which the taxonomist evidently treats as a confound, is instead the actual focus of studies under the rubric of growth allometry that are this paper's concern. Burnaby (1966) makes the claim even more 
explicitly: when variation due to growth is "extraneous to the desired comparisons," it serves solely as a "nuisance factor in morphometric work" that needs to be "eliminated" from diverse other multivariate methods for population comparisons. The method of "trend-surface analysis of transformation grids" that Sneath (1967) would introduce four years later turns explicitly to this issue of the "small number of underlying causes" for data sets involving multiple landmarks without, however, reopening this question of why we should be dividing out any summary second-moment quantity in the first place. We will shortly return to this concern for a better methodology of residualization in respect of size by altering the list of dimensions undergoing standardization to include an additional term from the lore of GMM, the uniform component.

In addition to this consistently overlooked intellectual aspect of the Procrustes approach, several anomalies in the approach to allometric analysis ascribed to "standard GMM" in the figures of the previous section are worth noting that this Boas method, the cancellation of scaling, neatly circumvents. The most fundamental of these anomalies arises from the conventional notion that any analysis that is to be called "allometry" must involve the separation of the data signal into two channels, "size" and "shape" in some paired sense. For instance, Sneath's great paper of 1967, which was one of two principal stimuli for my own dissertation a decade later (the other was Gould 1966), states at the outset, as an axiom requiring no defense, that once "corresponding points" have been marked on two or more images of organisms, "the diagrams are scaled and fitted to give the best possible fit; this gives measures of size and shape difference." Why "scaled and," and why "size and shape"? Corruccini's approach just reviewed likewise simply glides over this crucial decision, insofar as his interest is not in the growth of his specimens (the two species of hylobatids) but their distinction as species, given that he already knows Symphalangus is larger.

Writing at about the same time from a quite different disciplinary perspective, Gould (1966) embraces this same anomaly when, at the very outset of his argument, he says that he uses the term 'allometry'

in its broadest sense, to designate the differences in proportions correlated with changes in absolute magnitude of the total organism or of the specific parts under consideration.

But this is certainly not the "broadest sense" of this term, which had already been formalized by Huxley (1932) in his notation of power laws relating multiple size measures on the same organism. Gould may have been an accidental victim here of the syntax of the English language- the title of his article, "Allometry and size in ontogeny and phylogeny," suggests that "size" needs to be contrasted with something, whereas a title like "size and size in ontogeny and phylogeny" would be more consistent with the actual data analysis problem at hand; but a phrase like "size and size" seems very peculiar in English (although the same phrasal structure with a comparative adjective, "bigger and bigger," would not be). This is not a new point: see, for example, Bookstein (1989). In summary, a rhetoric of "size versus shape" is inappropriate for studies of growth per se.

Most of Gould's reflections on how to discuss issues of "allometry and size" concerned either qualitative descriptors of shape classes or else "proportions" construed as explicit decimal ratios between prespecified pairs of single quantitative variates. Only at the end of his article, Sect. VIII, did he broaden his scope to consider "total form alteration." In this context he recognized three categories of method: transformed coordinates sensu D'Arcy Thompson, "sets of allometric equations" such as growth gradients, and "multivariate analysis," here meaning the computation of principal components and their interpretation as factors in keeping with later expositions such as Blackith and Reyment (1971), Reyment et al. (1984), or Reyment and Jöreskog (1993). (The Boas method of the present essay would be categorized as a composite of the second and third classes here, growth gradients and multivariate analysis.) In any of these extensions of what was originally a bivariate rhetoric, the notion of a "proportion" (a single discrete ratio of particular interest) has vanished. Once the morphological complexity of a data set has risen toward our contemporary standard, no dichotomy remains between a "Gould-Mosimann school" and a "Huxley-Jolicoeur school" of biological interpretations (contra Klingenberg 2016) - the whole idea of "proportion" is an atavism of its origins in the language of Galileo's time, when notions of biometrics were indeed a novelty. For half a century now we have been well beyond that stage's focus on bivariate scatterplots and the corresponding simple linear regressions or residuals.

An even broader sense of this concept of allometry, hinted at by Gould's refusal to define what he meant by "absolute magnitude," involves the interaction between notions of the comparison of size measures vis-á-vis those of shape. When applied in studies comparing the sizes of anatomical substructures, the notion of "size" embraces not only quantities of root-mean-square type, such as the Centroid Size that the present article will compare to the least-squares-optimal score on Boas principal component 1, but also such familiar measures as perimeter and area, which, although appearing symmetric in their treatment of individual coordinates, nevertheless show enormous sensitivity to the actual shapes of the anatomical components they are intended to quantify. For example, a pair of four-landmark forms of which one is a square of side 1 and the other a rectangle of sides 0.129 and 3.871 have areas in the ratio of $2: 1$ but perimeters in the ratio of 1:2-if the space of alternative size measures is not delimited in some theoretically sensible fashion, the 
ratio description of allometry is uncertain even as to polarity. The ratio of Centroid Size measures for this pair of forms is even more extreme, more than 2.7 to 1 . This shape change is not intended to be biologically realistic, of course, but only serves to illustrate the algebraic pathology here. Jim Rohlf (pers. comm.) is fond of pointing out a similar problem that afflicts numerical taxonomy: classifications, clusterings, or cladograms based on different choices of a distance measure between taxa can yield entirely different estimated phylogenies. And the Procrustes world does not have access to any equivalent of the molecular-clock models attempting to ground such a choice in biologically meaningful quantities measurable by other technologies than imaging. Procrustes distance, in other words, should not be considered a biologically meaningful quantity, but only a human artifact along the lines of the sums of squares and crossproducts that appear in many other biometrical contexts.

Thus the words "size" and "shape" are not terms of art in morphometrics - they cannot be assigned operational meanings a-priori, but must be defined explicitly in the context of each study separately. In particular, for landmark-based growth studies, the division of the explanatory realm into two classes of reportage, "size" and "shape" without any algebraic notation, the way Gould does it, is not a scientifically productive strategy, separating as it does a multivariable signal homogeneous in units into two incommensurate channels (one in centimeters, one that is technically unitfree) whose relationship is too confounded a function of the unstated algebraic details of their measurement. Both the experimental programmes of Hans Przibram's Vienna Vivarium (sketched below in the Discussion) and the powerlaw models of Julian Huxley (1932) instead dealt with a multiplicity of length measures by explicit tabulations. The description of a data set of Boas coordinates by the centric allometry language of this paper can be considered a special type of these (see Appendix A.3). The biometrics of growth per se, as distinct from the biometrics of phylogeny, can go forward perfectly well as the biometrics of some laboratoryderived series of growing numbers - there is nothing intrinsically important about the space of their ratios, which is to say, geometric shape (see Bookstein 1986). ${ }^{5}$

An insistence on separating "size" from "shape" may have served the purposes of numerical taxonomy, which (forgive the oversimplification) aims more centrally at discovering groups than at classifying specimens into groups or at understanding functional morphology or morphogenesis within those groups. In Sect. 5.3.7 of Sokal and Sneath

\footnotetext{
5 This comment is not intended to impugn the importance of a list of specific formulas for ratios known a priori to be of biomechanical importance. Studies of this type, combining morphometric data with data from other disciplinary domains with corresponding data regarding function of the same animals, fall outside the scope of this article.
}

(1963), for example, the coefficients of an allometric relation are described as characters in their own right for studies in which the units are taxa rather than individuals and where size range per se is a less useful quantification. It is possible that this segregation of multiple centimeterscaled mensurands into two channels, "size" remaining in centimeters while "shape" has become unit-free, is serving a social, rather than a biological, purpose. The sociologist Bruno Latour argues in his Latour (1990) that ever since the invention of printed diagrams back in the sixteenth century one of their main advantages is the ease with which they can be combined with written text-turned into components of a book or a scientific article. Phelps (1932) may have been the first publication to do this explicitly when she experimented with diverse polygons of cranial landmarks (see her Figs. 2, $3,4,5,6)$. Then another way of justifying the rescaling step of an ordinary Procrustes analysis is the purpose of printing images of all specimens to the same scale on the page, as in the diagrams of Medawar's famous extrapolation of Thompson's method (Medawar 1945) to embrace specific comparisons of growth stages.

A different attempt to avoid rescaling, Todd and Mark's (1981a) method of "cardioidal strain," explicitly defended its purpose as psychological. Its authors, they say, "have recently demonstrated that radial transformations of human facial profiles [such as their "cardioidal strain" model produces] are generally perceived as growth by naïve observers ....whereas other classes of transformation ....are almost never perceived as growth." Ironically, I criticized the ToddMark article in the year of its publication for precisely that reason (Bookstein 1981) — the strain model there used only four coordinates of morphometric data out of the myriads afforded by the real human cranium; their "cardioidal" scheme was too simplistic to be of any analytic use. A reply by Todd and Mark, appearing in the same journal issue (Todd and Mark 1981b), retorted, in effect: let's see you do better. The present essay might indeed be seen as a belated constructive response to the mainly destructive critique presented in Bookstein (1981).

Assume that if "size" is to predict anything, the reification chosen for that construct should be the one that predicts the data as well as possible in a least-squares sense. But that is, according to the singular-value decomposition (an elementary theorem), a characterization of the first principal component of the data, the very formulation being diagrammed here as superimposed over the average form in Fig. $4 b$ and later analogues. Then the step in the Procrustes method that divides by Centroid Size is incompatible with the actual reporting of an allometric dependency except when the allometry that is the intended subject of discussion is in fact totally absent. Likewise, for authors who, like Sneath or Corruccini, are recommending a leastsquares method such as trend-surface analysis, why is the 
Fig. 6 Net assessment of the implications of the size standardization omitted by the Boas algorithm for the explanation of patterns of the resulting registered configurations. Top row: residuals from the first principal component of the Procrustes shape coordinates (left) versus those omitting the standardization by Centroid Size. Second row: the same after the uniform component has been partialled out from both systems, hence the descriptions "nonaffine nonallometric" of their panel labels (a truncation of "nonaffine nonallometric shape"). These two plots are indistinguishable
Procrustes, residuals from RW1

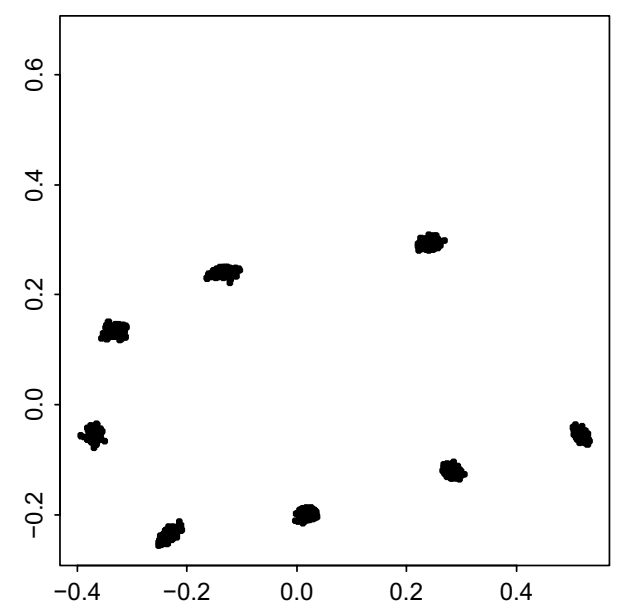

Procrustes nonaffine nonallometric

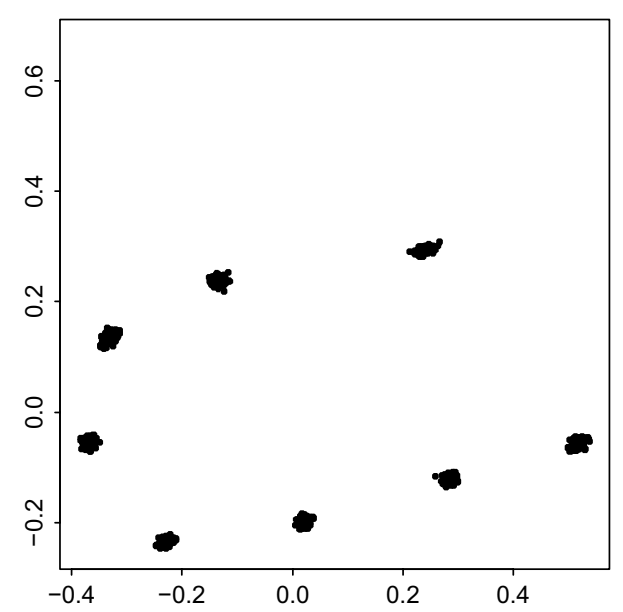

Boas, residuals from PC1

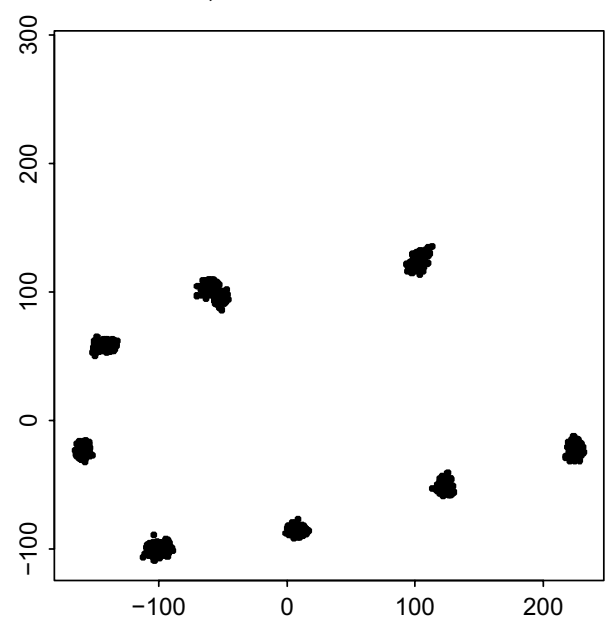

Boas nonaffine nonallometric

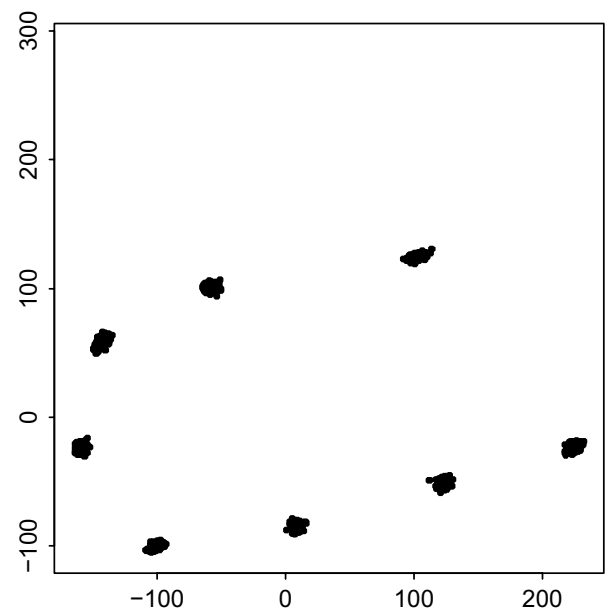

role of size taken as a divisor rather than a regressor? After all, in the principal-component approach, the second principal component (I will have more to say about this anon) is an analysis of residuals of the measured data after the effect of the first principal component is accounted for by ordinary linear least-squares; but a division by size is inconsistent with any least-squares residualization as examined at any later stage by principal components, analysis of variance, or anything else. Bookstein $(2014 ; 2018)$ has shown how to approximate division by size using an approximate linearization, regression-out of the differential of the explicit formula for Centroid Size (see Appendix A.2). But this does not matter for a methodology in which whatever "residual size-shape correlation" remains (the phrase is from Corruccini 1981) is adjusted out by linear regression of the shape coordinates on Centroid Size after that same size correlation has already been divided out in the course of the overall standardization. Evidently, in this approach size has been corrected for twice, once by division and again by regression. Such a procedure makes no sense.

How can we assess the net import of this double division? Consider the pair of comparisons in Fig. 6. In the top row, the scatterplot on the left is of residuals from Procrustes RW1 —of course, Centroid Size has already been divided out from these coordinates-whereas the scatterplot on the right is of the residuals from the Boas coordinates' PC1, without any division. Thus the righthand scatter has been residualized from only one regression (removal of PC1), whereas that on the left is the result of two separate standardizations, of which the first was, without any justification, carried out by division instead of by regression. In the lower row are the same scatters after a further standardization, this one removing the uniform component of variation (combination of shear and dilation, the transformations that leave all parallel lines parallel). The coordinates on the left are those of the standard Procrustes toolkit. Those on the right have been standardized case by case instead, by explicit fit of the Boas 
coordinate configuration on the coordinates of the average form separately in $x$ - and $y$-directions, hence, a fit that incorporates a standardization of the uniform component (including its aspect of scale) for each of the Cartesian coordinates separately, but by least squares, not by division. ${ }^{6}$ But these plots are almost exactly the same (except for scale). In other words, a three-step procedure-dividing by Centroid Size, then regressing out Procrustes RW1, then regressing out the Procrustes uniform term-is equivalent to the twostep procedure of regressing out Boas $\mathrm{PC} 1$ and then the Boas uniform term. Thus the original division by Centroid Size is irrelevant to the analysis of growth allometry for these data-it adds no insights beyond those that would have been available already by analysis of the Boas coordinates, which were only centered and oriented, not also rescaled. I return to this topic, the trade-off between geometrical notation and biometrical insight, in Sect. 4.

The approach to growth analysis recommended in this paper, already demonstrated in Fig. 4b, is least-squares in the original Boas sense, namely, with respect to the centered, reoriented coordinates of the original Cartesian data in their original metric units. It is important to keep in mind that this common center at $(0,0)$ is purely an algebraic artifact-I am not aware of any convincing method for validating this locus or any other "biological" allometric center by purely geometrical considerations. The only serious article I am aware of on this theme is Moss et al. (1983), which I reprogrammed for Fig. 5.55 of Bookstein (2018). Moss et al. discovered that the problem of finding a clear "center" of allometric growth of the human skull, meaning, a point around which variation of displacements was maximally radial rather than angular, had no good computational solution, and Bookstein (2018) concurred. (The next year Moss et al. (1984) replaced the centering scheme with a less constrained protocol, the "allometric network," that fell much closer to the original Huxleyan scheme of multiple interlaced power laws formulated in terms of interlandmark distances.) In another irony, Bookstein (1983) had already demonstrated that for any quadrangle of landmark locations in two forms there is an entire curve of exact candidates for the allometric center,

\footnotetext{
${ }^{6}$ In $\mathrm{R}$ this could be the command for (i in $1:$ nspec) boas. nonaff.x $[i]<$,- boas.mean.x $+\operatorname{lm}$ (boas.x $[i,] \sim$ boas.mean.x + boas.mean.y)\$resid where nspec is the count of specimens, boas.mean. $\mathrm{x}$ and boas.mean. $\mathrm{y}$ are the $x$ - and $y$-coordinates of the mean form, and boas. $x$ and boas. nonaff. $x$ are the usual matrices, specimen count by landmark count, for the Boas $x$-coordinates and their uniform standardization; followed by the analogous command for the Boas $y$-coordinates. (In the Boas approach, unlike the Procrustes, the calculation of the uniform component proceeds independently in the two Cartesian coordinates.) However, the two regressions together span only three degrees of freedom, not four-as a pair, they are constrained to have no net rotation around $(0,0)$.
}

a curve whose equation is cubic in the Cartesian coordinates, and for any two pentagons there is still, in general, a discrete set of exact solutions. The interpretation of diagrams such as that in Fig. 4b should thus be limited to aspects that can be put into words without explicit reference to that center.

These descriptions include interlandmark segments that, drawn as linear loci, pass close to this fictional center, and also segments between adjacent landmarks of a boundary, for which divergence of the vectors in Fig. $4 \mathrm{~b}$ can be interpreted circumferentially (as will be exemplified in Sect. 3). Interpretations like these are consistent with biological explanations in terms of interstitial growth, directional growth within the tissues in-between the landmarks. They have less a-priori cogency in applications to phylogeny, where such explanations have no corresponding explanandum-after all, nothing "grows" from Pan to Homo, whereas Vilmann's rodent neurocranial octagons did indeed grow from age 7 days to age 150 days. Hence the analysis in Fig. 4b should not be interpreted as if it mapped growth over the interior of the head-there is nothing biological about that center point; it is simply the superimposed centroid of all the given landmark configurations. The next section returns to this theme via experiments with truncation of landmark lists in the pursuit of more and more convincing Boas growth analyses.

Figure 7 helps us explore the implications of the new method for this specific data set of rodent neuroanatomical octagons. Figure 7 a compares the two different estimates of "size" arising from our two different GMM protocols, Centroid Size (horizontal) versus BPC1 score (vertical), as applied to Vilmann's rat octagons. These two measures correlate nearly 0.999 , and both are in units of length, but only the Boas version incorporates (via its loadings) the actual effect profile of size as an allometric factor, the presumptive deliverable of a growth allometry analysis. Although the two size variables correlate nearly perfectly, they have radically different formulas. One of them, Centroid Size, has a formula that pays no attention to actual sample variation-it is merely a multiple of the root-mean-square of interlandmark distances case by case-whereas the other candidate, PC1 of the Boas coordinates (BPC1), is calibrated to the actual covariance structure of the Boas coordinates, thereby capturing much of the effect of growth allometry that was the focus of Vilmann's actual experimental study. These coefficients are displayed explicitly in the dashed lines of Fig. 4b, each one an $(x, y)$ pair for the corresponding pair of loadings in the actual formula for this PC1. (The drop in this size-size correlation from this figure to some of those in the next section will be part of the argument that the data there need subsetting prior to any study of growth allometry per se.)

Continuing, Fig. 7b, c show the vertical components of the two panels in Fig. 3 as thin-plate splines of their own. The grids agree regarding the posterior shear of the roof of the calva with respect to the cranial base, but only the Boas 


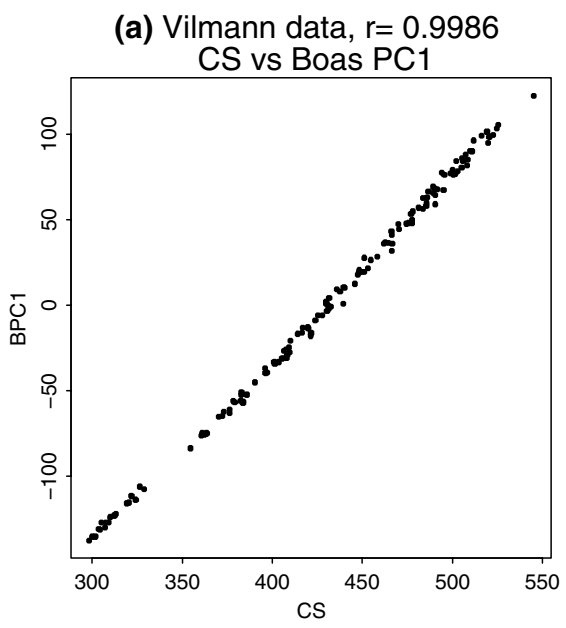

Fig. 7 Additional aspects of the Vilmann analysis. a Scatter of the alternative size measures, Centroid Size (divisor in the Procrustes method) versus principal component 1 of the Boas coordinates. b, c

version also shows the nonlinear change in proportions along the cranial base and the contrast of SOS-ISS and Brg-Lam growth rates. Still, because division by Centroid Size is so nearly equivalent to a projection, the scatter at upper left in Fig. 6, Procrustes shape residualized on RW1, is very nearly the same as the scatter below it, which is further residualized on the uniform term. Note (Fig. 1) that this is not merely because growth is "nearly uniform"-in both analyses there is clearly a strong nonuniform (nonaffine) component that combines a growth-gradient, a multiple of partial warp 1, with a focal effect between Opi and IPP (see e.g. Bookstein 2018, Figs. 5.84, 5.98). Analyses in which PC1 is to be interpreted as "general size" clearly need to be of the Boas coordinates, not the Procrustes coordinates from which size (in the sense of Centroid Size) has already been divided out.

The more cogent defense of the appearance of Centroid Size in the geometric morphometric context pertains to its formula as a score, not its use as a divisor. As Bookstein (1986) showed heuristically and then Dryden and Mardia $(1998,2016)$ showed analytically, one particularly symmetric family of statistical models for the Cartesian data (the "isotropic Mardia-Dryden," independent circular normal variation at every landmark around some fixed template), which is spherically Gaussian in its space, yields an almost exactly spherical distribution of the Procrustes (but not the Boas) coordinates in their space. On this model, furthermore, the covariance of Centroid Size with every shape measure (that is, with every direction in this shape or form space), in the limit of indefinitely large samples with infinitesimally small shape variance, is exactly zero. This means that on the assumption of a wholly isotropic distribution of landmark coordinates around a shared template, one can examine an allometric relationship for "statistical
Thin-plate splines of the second components in the scatters of Fig. 3 of which the first components were already displayed in Figs. 1 and 2

significance"-for a correlation of landmark proportions with size — by calculating the dependence of Centroid Size on the list of all the shape coordinates (in practice, its multiple correlation).

The extension to using this score as a divisor may arise from simple graphical convenience (the desirability of being able to represent configurations being compared in panels of approximately the same size on the printed page, regardless of details of their shape) or from the fact that the formal linearization of the Procrustes procedure, the $J$-matrix notation of Appendix A.2, shows that for size to be used to augment a list of shape coordinates in a way preserving the spherical symmetry of the composite, it is necessary to replace Centroid Size by its logarithm, the differential of which is scaled as the reciprocal of size per se around its own sample mean. With this transformation, in the limit of small variation, the variability of Centroid Size is commensurate with that of any other explicit dimension of shape variability if formulated in a linear notation at unit Procrustes length under the same Mardia-Dryden model. But the symmetry of this model makes it essentially valueless for biometrical applications, where in virtually all contexts the distribution of landmark Cartesian coordinates is not symmetrical in that pervasively spherical way (Bookstein 2016). Then the justification in terms of division does not apply to the data likely to arise in any biological application; in any event, the formulation of the Procrustes algorithm, with its culminating division by size, came many years before the Mardia-Dryden formalism.

Let me summarize the argument to this point. The standard Procrustes procedure (for which see the standard GMM textbooks) incorporates a step, the normalization of geometric scale using the formula for Centroid Size, that appears 
animal 1

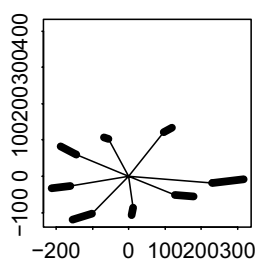

animal 6

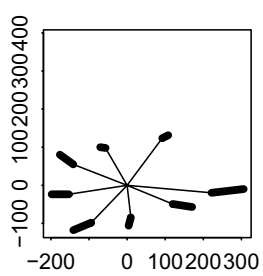

animal 11

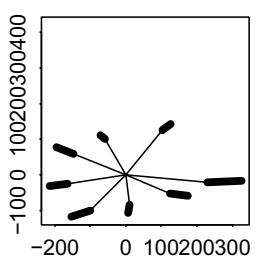

animal 16

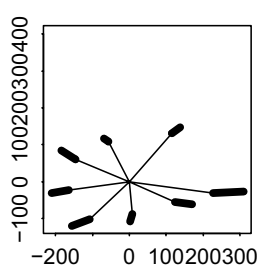

animal 2

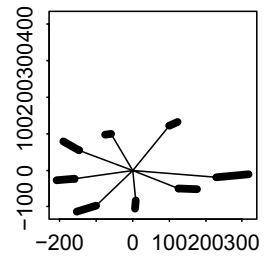

animal 7

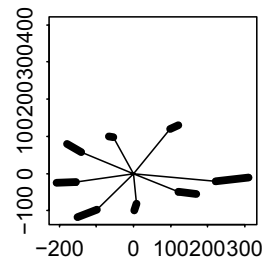

animal 12

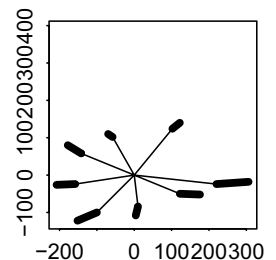

animal 17

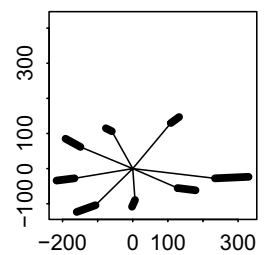

animal 3

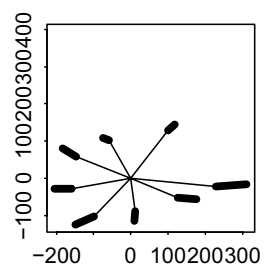

animal 8

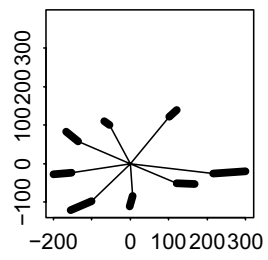

animal 13

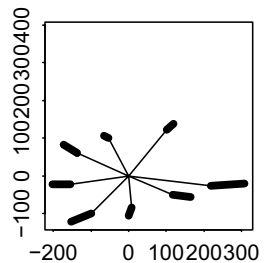

animal 18

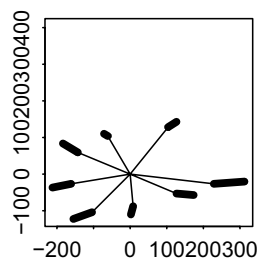

animal 4

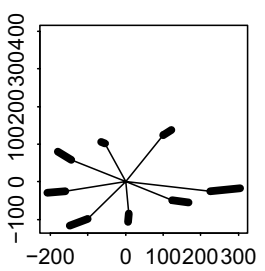

animal 9

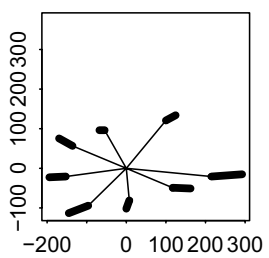

animal 14

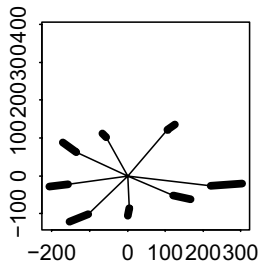

animal 5

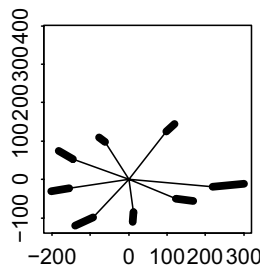

animal 10

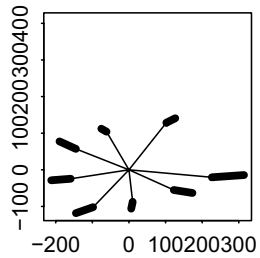

animal 15

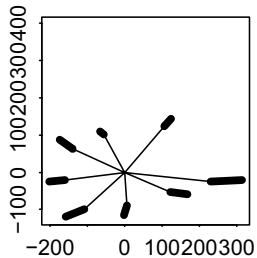

Fig. 8 Replication of the centric allometry analysis, Fig. 4b, for each of the 18 experimental animals individually. The thick lines replicate, for each animal separately, the same vectors that were represented by dashed lines in Fig. 4b. The separate panels are indistinguishable except by tiny details of these growth trajectories

The Vilmann data set may not be a representative example of allometric studies - after all, it represents the closest that that competent mammalian experimentalist could come to a repeated measurement of the selfsame growth process. A design nearly as effective is the "longitudinal growth study" that is traditional for the best human craniofacial work (cf. Riolo et al. 1974). But most studies of allometry, following Gould (1966), instead involve "crosssectional" samples, observations of multiple organisms at one single time. Paleobiological studies, in particular, are necessarily of this design. For the resulting inferences to have any validity it is appropriate to ask to what extent the analysis of such a design, in which every organism's ontogeny is calibrated to its own environment, matches the analysis of any representative individual had it been the sole specimen under study. 
By virtue of its perfectly balanced longitudinal design the Vilmann data set permits us to examine this question explicitly for the subsample of 18 animals with complete data that this paper has relied on. Figure 8 replicates the analysis of Fig. 4b separately for each of the 18 rodents in the Vilmann study. For ease of viewing at this reduced scale, the lines that were dashed in Fig. $4 \mathrm{~b}$ here are thickened instead. Animal-to-animal variability here, while detectable by careful scrutiny, is evidently insubstantial. The apparent invariance of growth trajectories in Fig. 8 is across the full age span of the animals imaged. (For a finer-grained representation, age 7 days to age 14 days only, see Fig. 7.20 of Bookstein 2014.) In other words, the centric allometry we are seeing is not an artifact of averaging over diverse individuals, nor is it an artifact of the strength of the overall correlation of size with shape; instead it is a characteristic of the growth trajectories separately. (In cladistic terms, it would be called a character in its own right.) The invariance in this figure thus suggests a mastery of the experimental setting completely in keeping with the idealizations of one philosophy of biology, the founding of morphological explanations in actual morphogenetic data under the tightest possible experimental control (Vilmann's animals were "close-bred"). An analogous study in human children would involve careful serial imaging of multiple sets of monozygotic twins (replacing experimental control of embryonic conditions by explicit identity of genotypes). But I am aware of no such data set in the craniofacial literature.

\section{An Example from Anthropoid Phylogeny}

The degree of experimental control of animal material demonstrated by this last analysis of the Vilmann rodents is far from typical for the sort of data sets one encounters in metazoan evolutionary biology. It is much more usual to be dealing with data that is heterogeneous as regards ontogeny, phylogeny, or both. This section offers a provisional guide through a centric allometric analysis for contexts like that. The key principle will turn out to be subsetting both of the landmark data facets at once: not only the specimen list but also the landmark list. The ultimate purpose of such a workflow is analogous to the purpose of random-walk modeling in paleobiology (see, e.g., Bookstein 2012): to extract a particularly simple model the residuals from which have a seemingly unpromising structure not worthy of further investigation in any detail, and then to speculate on why the living form has aligned with this particular model. In our context of geometric morphometrics, such a desideratum, arrival at a representation of residuals that justify ceasing to analyze further, is typified by the lower row of diagrams in Fig. 6. Such diagrams resemble the corresponding diagrams of the (overly symmetric) isotropic Mardia-Dryden distribution involving independent, identically distributed circular noise at each landmark of a common template (see Bookstein 2018, Fig. 5.37 or Dryden and Mardia 2016, Sects. 11.1.1-11.1.2). Whether there is a relatively weaker residual factor structure in such scatters considered multivariately is not as relevant as their greatly restricted variance compared to the variances by landmark which which they began. That is, the point will be the replacement of the variation in Fig. 2a by the variation in the lower row of Fig. 6 , further investigation of which in the data set at hand would not be a fruitful use of the biologist's time.

An exemplary "realistic" demonstration begins, then, with the customary announcement of a template for the anatomical characterizations of the landmarks that compose it. We already saw one of these in the diagram at the top of Fig. 1. The present Fig. 9, modified from Bookstein (2018), serves this function here: a combination of caricatures of the landmark points in situ with their Latin names and terse operational definitions. I cannot reconstruct any explanation of how this particular sample of 29 forms came to be delineated back in its original version of 2003-it is not a representative sample of any population of museum-accessible samples, available hominids, anthropoid taxa, or any other convenient rubric. Nevertheless it ends up serving a very useful pedagogic purpose as workbench for a demonstration of how to simplify anatomically complex extended landmark configurations by a combination of specimen sequestration and landmark sequestration.

From the most up-to-date prior analysis of these 29 20-landmark configurations, represented in my two textbooks (Bookstein 2014 and Bookstein 2018), it is helpful to extract the summary panels in Fig. 10. As this figure will be a model for the five that follow it, it is worth taking some space to explain its panels separately. The twenty landmarks are those of our template, Fig. 9. The specimens comprise 17 adult modern $H$. sapiens, 4 juvenile sapiens aged 2, 4, 4 , and 10 years, one archaic specimen (the one conventionally known as "Mladeč"), four H. neanderthalis (Atapuerca, Kabwe, Guattari, and Petralona), the single australopithecus specimen STS5 ("Mrs. Ples," although its sex is now considered uncertain), and one each of each sex of Pan troglodytes. The corresponding Procrustes scatter, Fig. 10a, shows considerable heterogeneity (though not to an extent beyond those of other paleoanthropological data sets in my experience), especially at Brg and Alv.

The first summary graphic in the conventional workflow for such a data analysis, Fig. 10b, is a projection of the first two relative warp scores of these 29 specimens (their principal coordinates with respect to Procrustes distance, square root of the summed squared landmark shifts in Fig. 10a). It is evident that this sample will not submit to any holistic multivariate investigation if this is how it looks in a preliminary exploratory analysis. In this RW1-RW2 plane the adult 
sample average positions

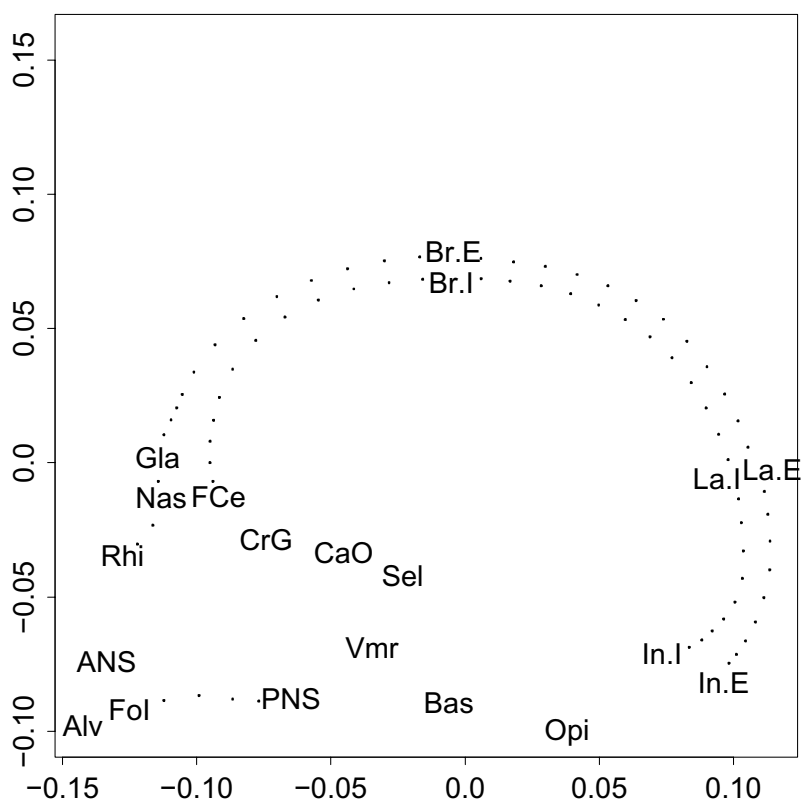

Fig. 9 Template for the data sets of up to 20 landmarks analyzed in Fig. 10 through Fig. 15. The data are sketched here as they might trace a typical form of the sample (in the original study, Bookstein et al. 2003, these were synthetic midsagittal images derived from CT scans). Landmark names and operational definitions: Alv, alveolare, inferior tip of the bony septum between the two maxillary central incisors; ANS, anterior nasal spine, tip of the spina nasalis anterior; Bas, basion, midsagittal point on the anterior margin of the foramen magnum; BrE, BrI, external and internal bregma, outermost and innermost intersections of sagittal and lambdoidal sutures; $\mathrm{CaO}$, canalis opticus intersection, intersection point of a chord connecting the two canalis opticus landmarks with the midsagittal plane; $\mathrm{CrG}$, crista galli, point at the posterior base of the crista galli; FCe, foramen caecum, anterior margin of foramen caecum in the midsagittal plane; FoI, fossa incisiva, midsagittal point on the posterior margin of the fossa incisiva; Gla, glabella, most anterior point of the frontal in the midsagittal; InE, InI, external and internal inion, most prominent projections of the occipital bone in the midsagittal; $\mathrm{LaE}, \mathrm{LaI}$, external and internal lambda, outermost and innermost intersections of sagittal and lambdoidal sutures; Nas, nasion, highest point on the nasal bones in the midsagittal plane; Opi, opisthion, midsagittal point on the posterior margin of the foramen magnum; PNS, posterior nasal spine, most posterior point of the spina nasalis; Rhi, rhinion, lowest point of the internasal suture in the midsagittal plane; Sel, sella turcica, top of dorsum sellae; Vmr, vomer, sphenobasilar suture in the midsagittal plane. Dotted curves sketch the alignment of these anatomical boundaries between the landmarks; once standardized, the dots turned into semilandmarks in a later version of this same data set explained in Sect. 5.5.4 of Bookstein (2018)

Homo sapiens form one cluster falling in-between a sparser cluster for the neanderthals and a separate string of three loci for the juveniles. Oblique to this collineation and more extended than the contrast between juvenile sapiens and neanderthals is a jet of points in order of their phenetic distance from Homo: first, the australopithecus; then the female chimpanzee; and finally the male chimpanzee, apparently a victim of his own hypermorphosis. As the Bookstein textbook narratives have already argued, such a plot indicates that the pooled analysis per se will not have any chance of making sense: the assumptions permitting an inspection of the RW's as coherent descriptive composites are, without exception, contradicted.

This inference is extended by the panels in the center of this figure. Panel (c), upper center, is the scatter of Boas coordinates this paper is recommending replace the Procrustes coordinates. In this Boas scatter, the larger forms are at greater distance from $(0,0)$ than the smaller forms, leading to an elongation of the distributions for some of the peripheral landmarks. The effect of this nonscaling is much clearer in panel (d), lower center: the replacement of the apparently directional RW1 of panel (b) by the pair of components here, nearly equal in explained variance, and for which the implicit dimension of growth (contrast of the juvenile $H$. sapiens with the adults) lies in a direction nearly along the second principal component rather than the first, where it would be expected to fall were this a legitimate growth study at this stage. In keeping with the panel to its left, the neanderthals still bracket the adult sapiens at one extreme, with the juveniles bracketing it at the other. But, in contrast to panel (b), the two chimpanzees now agree in their separation from the sapiens (while the male still deviates from the female in a direction that would again generally be taken to connote hypermorphosis). The grid for BPC1, panel (f), is shown here only because it will be in this position in each of the next five figures. But for this particular data set, 29 forms by 20 landmarks, BPC1 is irrelevant to any explication of growth allometry. One sees that pretty clearly as well in panel (e), the diagram that would show centric allometry if this data set behaved anything like the Vilmann data in Fig. 4b. Unfortunately, the net impression here is of something entirely different, a sort of spiderlike squashing whereby as many of the extreme landmarks shift obliquely to their radii as along it. The centric model, in short, is worthless for this sample. If we needed a numerical diagnosis of the problem, it is sufficient to compute the correlation between Centroid Size (the Procrustes scaling factor) and the first Boas principal component score, which is, as we explained above, the optimal explanation of all the landmark positions in a least-squares sense. This correlation is 0.849 , versus 0.9986 for the analysis of the Vilmann data in Figs. 1 and 2.

The display in Fig. 10b mandates that we discard the three outlying specimens-the three non-Homo specimensfrom the sample, leaving the 18 adult sapiens (including Mladeč), the four juveniles, and the four neanderthals. The corresponding six-panel display, Fig. 11, now looks entirely different, not so much regarding its coordinate scatterplots (panels a and c) as in its ordination plots by interspecimen distance (panels b and d). Both panels continue to show the 

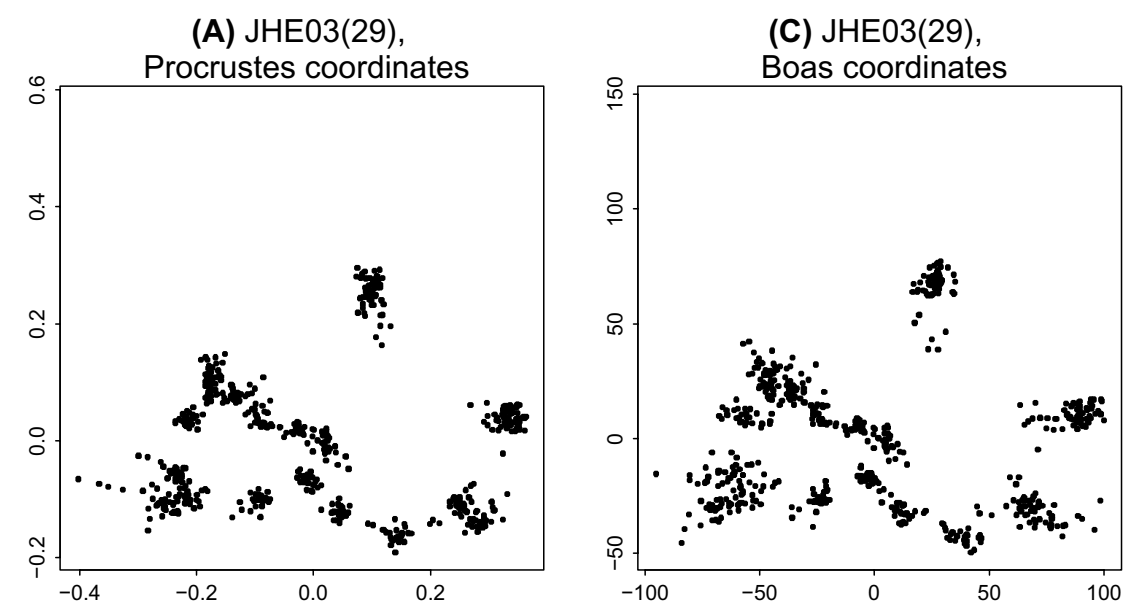

(E) Boas PC1 versus mean form (same scale)
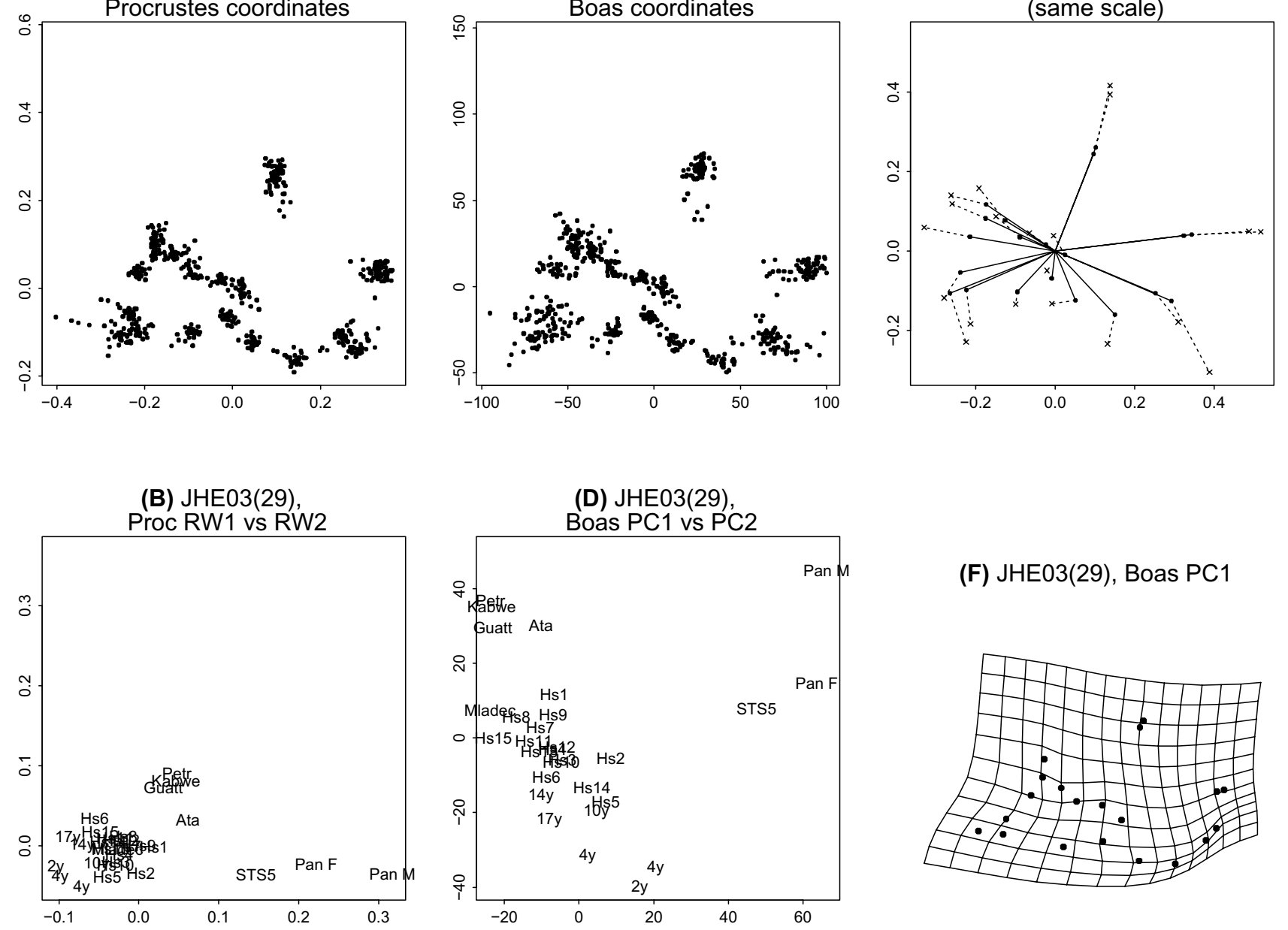

(F) JHE03(29), Boas PC1

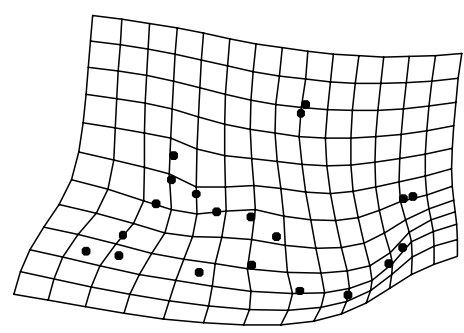

Fig. 10 Key frames of a dataflow of specimens digitized using the Fig. 9 template and then analyzed by the method of centric allometry described in this article. This analysis was of all 29 specimens using all 20 landmarks. Panels, here and in the subsequent five figures: a scatter of Procrustes shape coordinates; $\mathbf{b}$ scatter of Procrustes RW1 against RW2 scores (the first two principal coordinates of the summed squared differences of the coordinates in a); $\mathbf{c}$ scatter of Boas coordinates; d scatter of Boas PC1 against PC2 scores (first two principal coordinates of c); e centric allometry diagram for Boas PC1; f thin-plate spline for a visually suitable multiple of Boas PC1. For the relation between principal components and principal coordinates, see Bookstein (2018) alignment of the two four-specimen subsamples (juveniles at one end, neanderthals at the other) to either side of the central cluster of $18 \mathrm{H}$. sapiens, but now, with the other genera omitted, this axis determines the first principal component of the data quite precisely. And furthermore its precision is much greater in the Boas analysis than the Procrustes analysis, inasmuch as a strong correlate of this growth scaling, namely, Centroid Size, had been divided out in the course of the Procrustes analysis, greatly weakening its influence on the variance of the first principal component. The correlation of CS and BPC1 score has now jumped to 0.981. (But it will crawl even higher before we are done.)

Panel (f), the first Boas principal component as a grid, is familiar from decades of thin-plate-spline-rendered anthropometry. The neural skull shows negative allometry with respect to the facial skull, and while that neural skull deformation is close to isotropic, growth in the maxilla is highly directional (the trait usually called prognathism). The centric allometry diagram for this data subset, panel (e), no longer looks like a squashed spider with legs splayed every which way, but instead shows radial dilation throughout most of the maxillary landmarks. The alignment of the PC at Bregma, though, is azimuthal, not radial, and the discrepancy between the implied displacements at inner and outer inions is likewise incompatible with the underlying centric intuition. Finally, something looks perplexing at FCe.

So now it is reasonable to subset the landmark list, not the specimen list, by deleting the two Bregmas, the two Inions, and, because they are on the suture joining the bones on which this pair of structures lies, the paired Lambdas. (It 

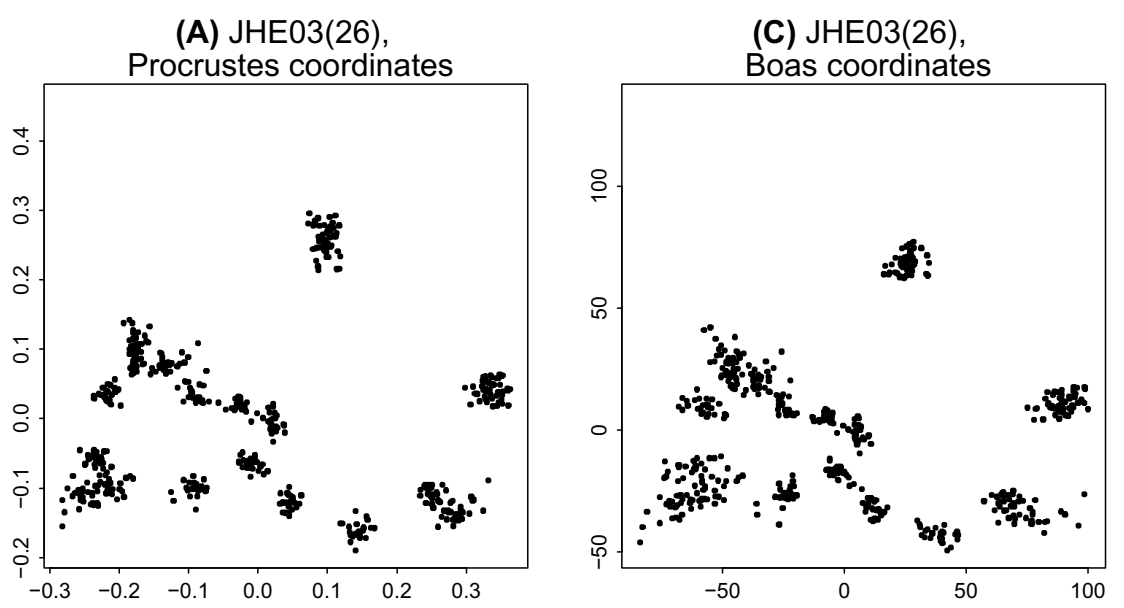

(E) Boas PC1 versus mean form (same scale)

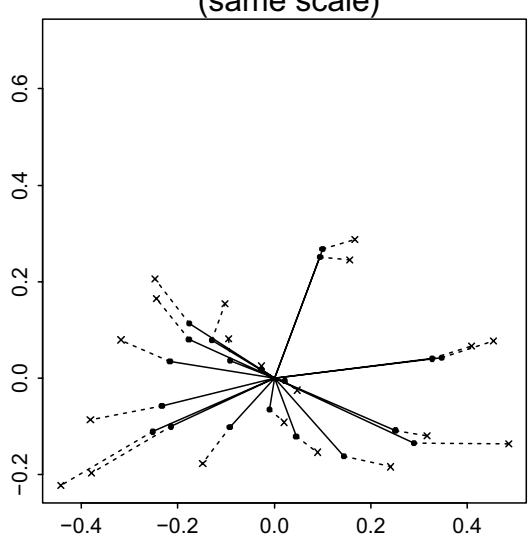

(F) JHE03(26), Boas PC1

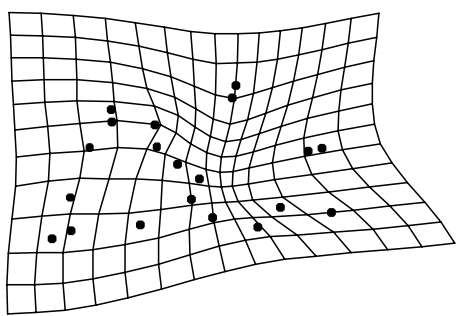

Fig. 11 Key frames of an analysis of 26 specimens, 20 landmarks as in Fig. 10

is possible that none of these are actually valid landmarks. Bregma, in particular, is known to sometimes arise as a wormian bone sliding along the dura over the course of early development. An extension of the centric method that would allow such points to slide along outline curves to a position of maximum consistency with the behavior of the remaining landmarks is imaginable but falls outside the scope of this paper, as a special case of a deeper question: whether the hominid neurocranium can be persuasively analyzed by landmark-based methods at all.)

The resulting analysis, Fig. 12, shows considerable progress. Limited to maxillary structures and the cranial base, the Boas ordination (panel d) is now even more consistent with a single dominant factor for allometry, while the landmarks of the centric allometry diagram, panel (e), now conform except at $\mathrm{FCe}$ and $\mathrm{CrG}$. The corresponding thin-plate spline, panel (f), looks just like the homologous sector of the more extended grid in Fig. 11. The correlation between CS and the BPC1 score is now 0.994 .
Neanderthals were a different species from us, and, furthermore, the present sample includes no neanderthal juveniles. (Research out of the Max Planck Leipzig may have obviated this problem, someday permitting a centricallometric analysis of a mixed sample of the two species of Homo in a balanced design. See Gunz et al. 2010 or Neubauer et al. 2018.) So it is reasonable to delete the neanderthals from this experimental data set at this stage. There results the analysis in Fig. 13, a sample now reduced to 22 specimens on the same 14 landmarks.

The scatters in panels (a) and (c) are much tamer now than they were in Fig. 12-the neanderthals are, after all, a different species - but this improvement in the taxonomy has had the unfortunate effect of completely destroying the interpretability of Procrustes RW1, panel (b). (This problem is typical, of course, of situations in which one applies a principal component analysis to a mixture of multiple groups: see Bookstein (2018), Fig. 4.3. The juveniles now comprise one corner of a plot on two axes of nearly equal variance, 

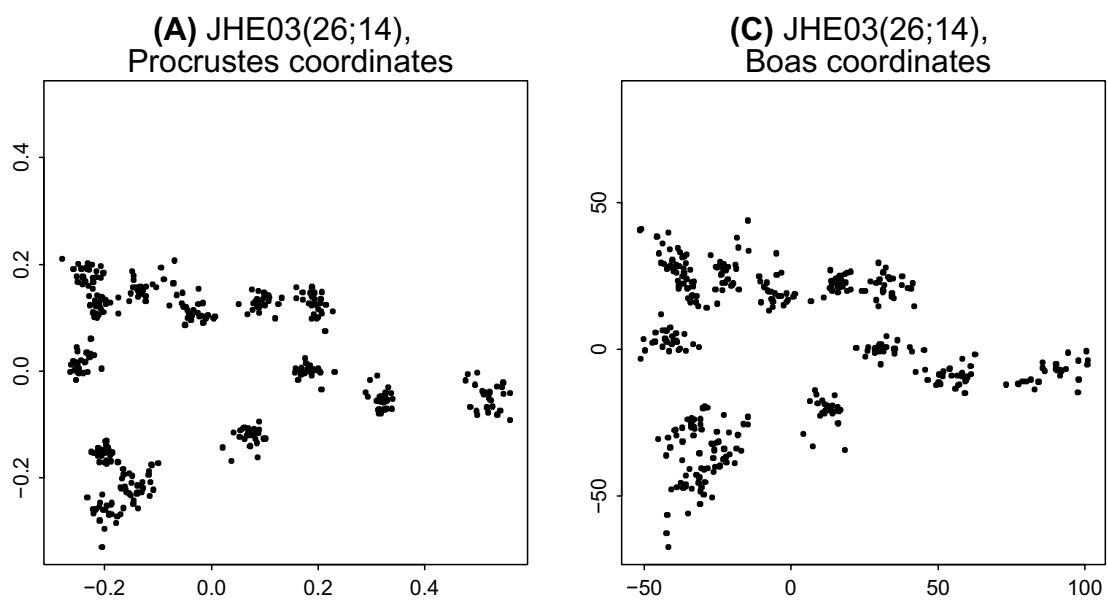

(E) Boas PC1 versus mean form

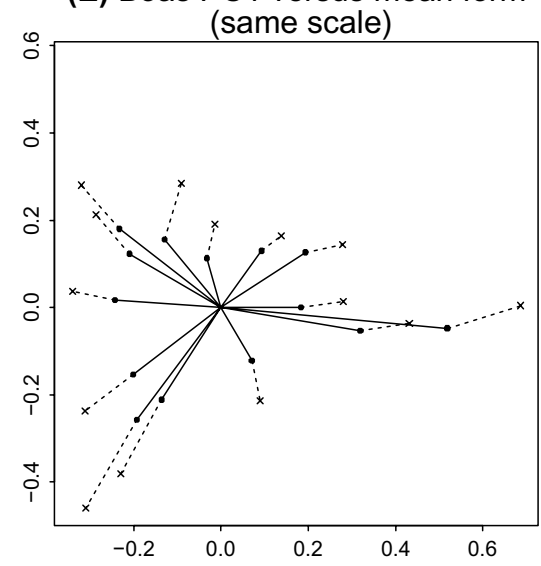

(B) JHE03(26;14),

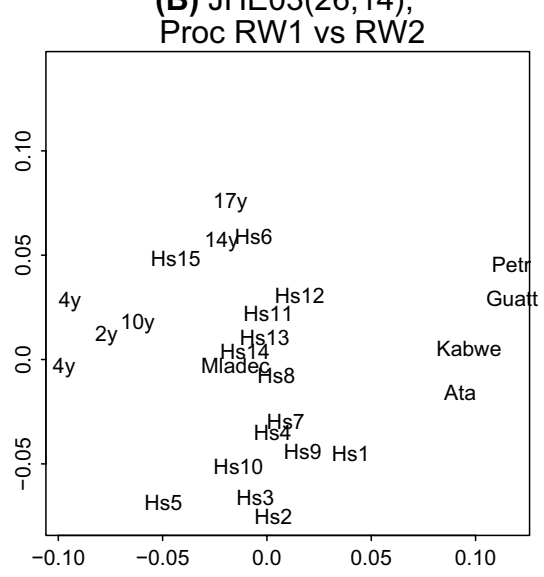

(D) JHE03(26;14),

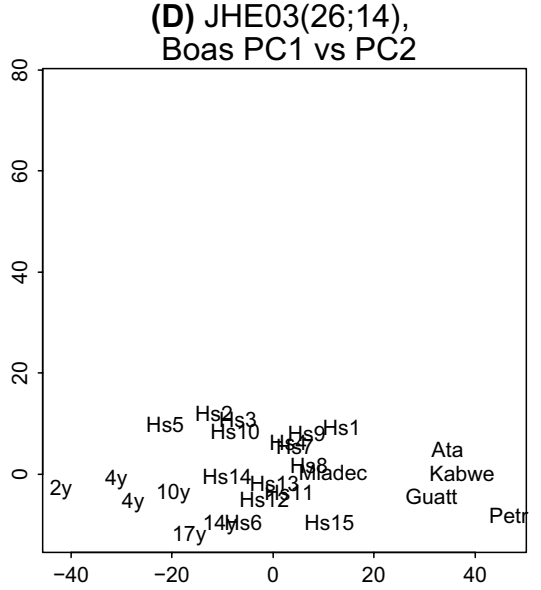

(F) JHE03(26;14), Boas PC1

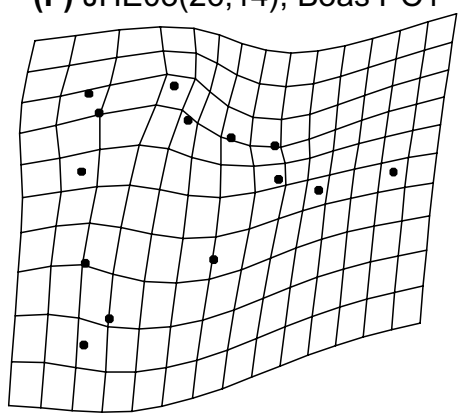

Fig. 12 Analysis of 26 specimens, 14 landmarks as in Fig. 10. Note that with the deletion of six landmarks the orientation of the mean form in a, $\mathbf{c}, \mathbf{e}$, and $\mathbf{f}$ has rotated by about $30^{\circ}$ on the page

with the remaining 18 sapiens distributed over both of the opposite edges.) The corresponding Boas ordination, however, has no such difficulty: the three youngest juveniles fall at one end of an obvious principal component whose other end combines the Mladeč specimen with two of the largest modern specimens. The centric plot, panel (e), remains fully radial except at $\mathrm{FCe}$ and $\mathrm{CrG}$, and the grid diagram, panel (f), is likewise unchanged in showing hypertrophy of the maxilla in a primarily anterior direction (meaning, anatomical anterior, which is rotated a bit downward of the printed horizontal in this Procrustes pose). And the correlation between Centroid Size and the BPC1 score has risen just a bit, to 0.995 .

The last two centric descriptions have both included the phrase "except at FCe and CrG." It is time to delete these two landmarks as well, thereby reducing our analysis to the maxilla along with the lower border of the cranial base (only). Anatomically, we have left the brain case; the analysis is now wholly limited to a combination of the cranial base and the face.
This adjustment (Fig. 14) clarifies the nature of the centric model without substantially altering any aspect of the actual arithmetic. The Procrustes ordination, panel (b), is still unusable, while the Boas scatter is barely changed from that of the previous analysis on 14 landmarks instead of 12 . The centric plot, panel (e), is now wholly radial, with a corresponding surge in that CS-BPC1 correlation to the satisfyingly high value of 0.997 . The thin-plate depiction of the growth factor, panel (f), is likewise unchanged between the panels except in the immediate vicinity of where those two deleted landmarks had been.

This would normally constitute the end of the data subsetting cascade. But for pedagogic purposes it is helpful to reverse the penultimate decision, the deletion of those four token neanderthals, to see how we are doing, at least in respect of the form of this species as adults. There results one final tableau of panels, Fig. 15.

With the neanderthals restored to the sample, the Procrustes scatter in panel (b) is not quite as unpleasant as it was in Figs. 13 and 14. The extra weight of those four additional 
(A) JHE03(22;14),

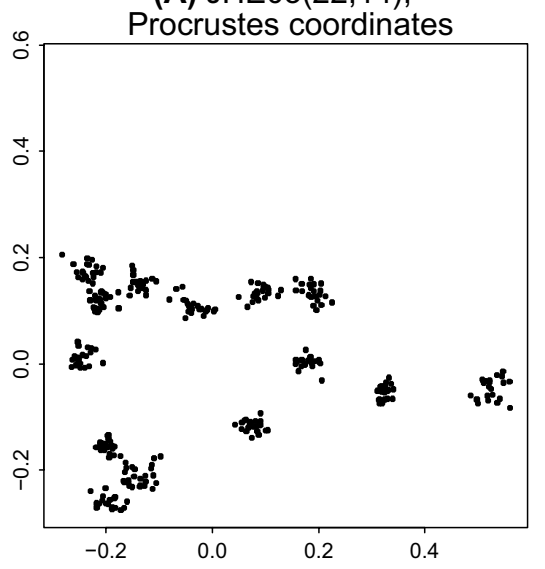

(B) JHE03(22;14),

Proc RW1 vs RW2

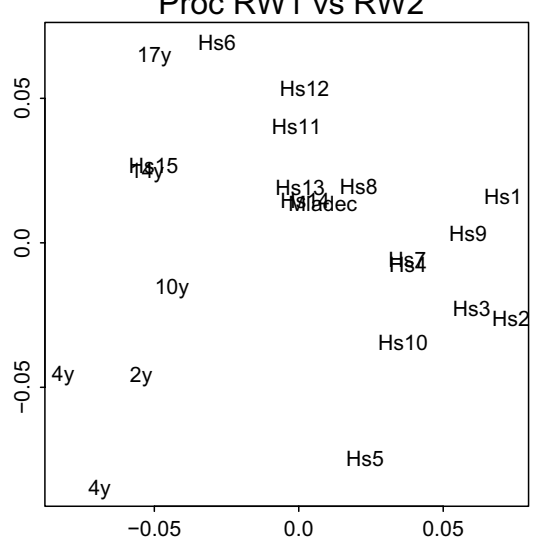

(C) JHE03(22;14),

Boas coordinates

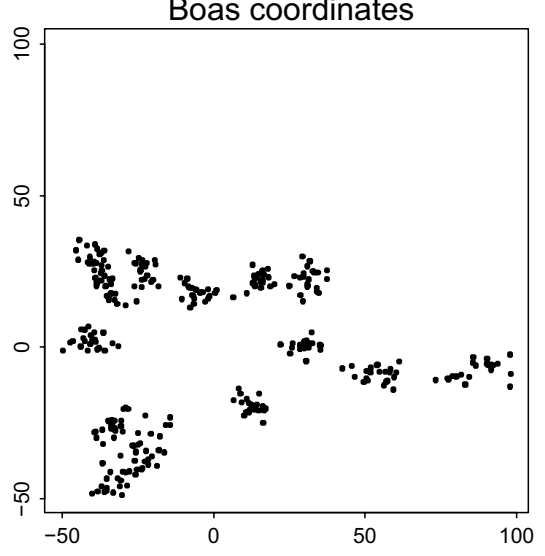

(D) JHE03(22;14),

Boas PC1 vs PC2

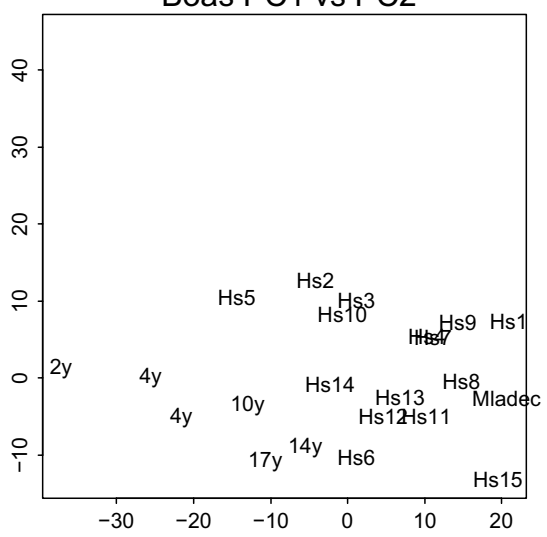

(E) Boas PC1 versus mean form (same scale)
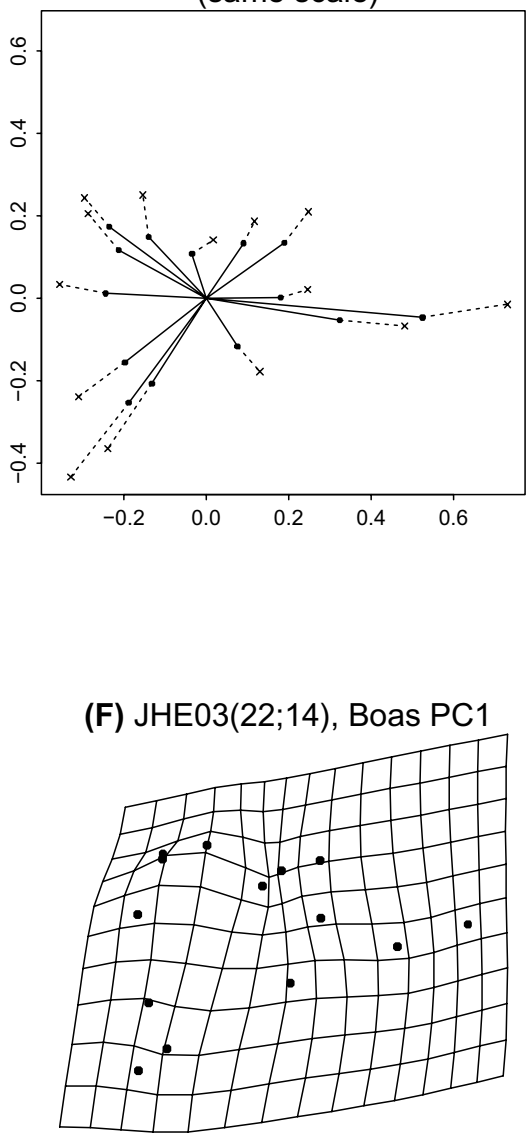

Fig. 13 Analysis of 22 specimens, 14 landmarks as in Fig. 10

specimens has the effect of rotating RW1 back onto the orientation it had in Fig. 12, with the sapiens juveniles and the neanderthals bracketing a big cluster of adult sapiens along the abscissa. But now the corresponding Boas plot, frame (d), is exactly what we would wish to see: the same description, but with the dominant axis of variation now very precisely specified with respect to the best alternative (the second principal component). The thin-plate diagram, panel (f), continues to clearly show the contrast between cranial base size and a maxillary size enhanced in the anatomically anterior direction, while the centric allometry plot, panel (e), remains reassuringly radial almost everywhere, consistent with this panel in the preceding figure. The correlation between Centroid Size and the first Boas principal component score is almost unaffected, at 0.996 , by this extension to an additional species (however poorly sampled).

For purposes of summarizing the power of the explanatory growth factor at which we have arrived it is worth examining one final display (Fig. 16) that shows all the variation remaining after we have regressed out BPC1 of Fig. 15 along with the uniform component of these forms (as explained in Sect. 2, Fig. 6 and footnote 6). Over the remaining twelve landmarks, these residual scatters look roughly circular and of comparable variance landmark by landmark. They are not independently distributed, but any noncircularity they might display is much less than that of the original Boas coordinates, Fig. 10c, nearly $80 \%$ of the variance of which they have exhausted by this pair of algorithmically rigorous least-squares maneuvers.

The example here should not be considered as dealing with any sort of "modularity." A landmark subconfiguration whose growth changes can be summarized by one single centric allometry does not thereby attain the status of any sort of developmental "module"-the explanation of this association could instead be relevance to a common function, biochemical pathway, or environmental constraint. Vilmann's purpose, like that of Melvin Moss (who first introduced the world of craniometrics to these data when he digitized them from Vilmann's original cephalograms), was to found an analogous methodology for the assessment of 

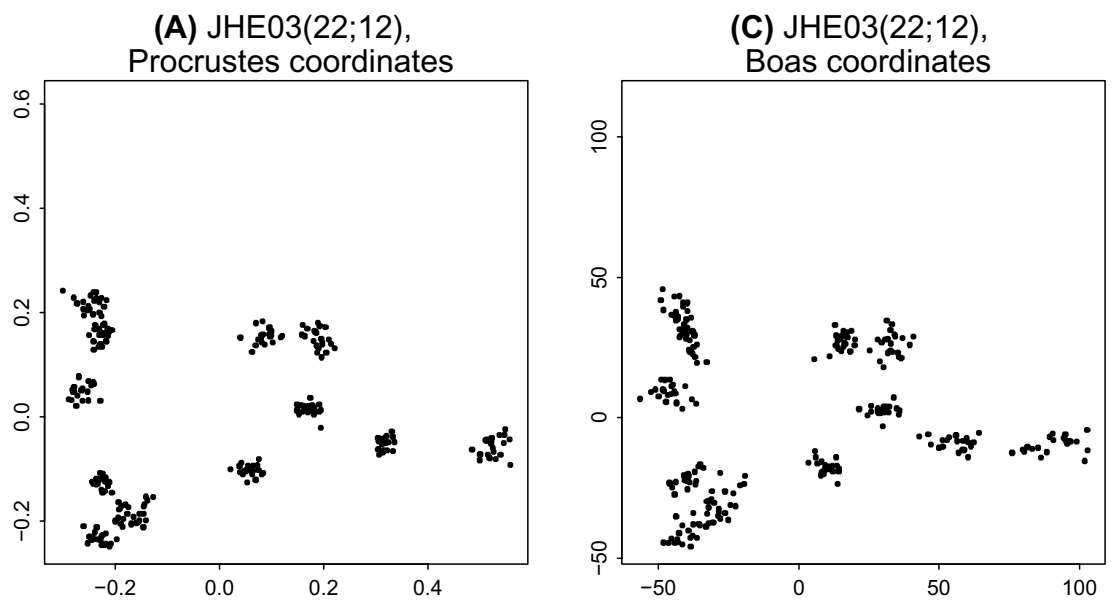

(E) Boas PC1 versus mean form (same scale)

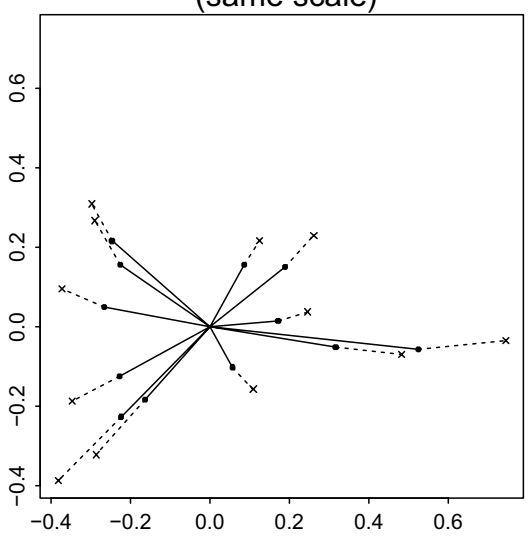

(B) JHE03(22;12), Proc RW1 vs RW2

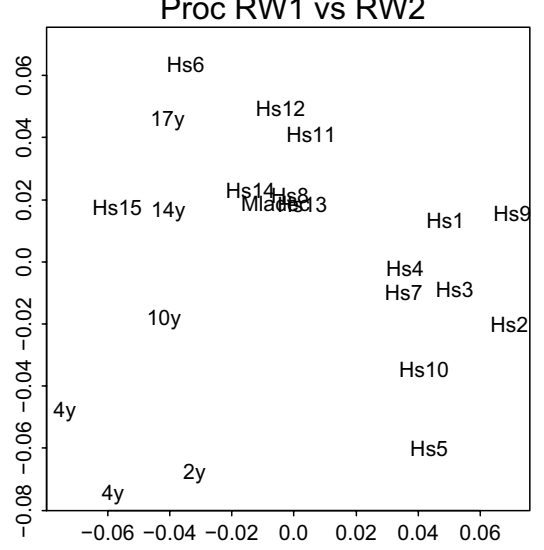

(D) JHE03(22;12),

Boas PC1 vs PC2

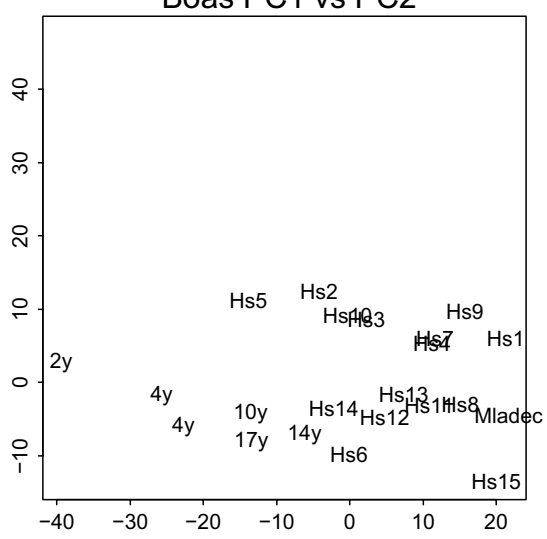

(F) JHE03(22;12), Boas PC1

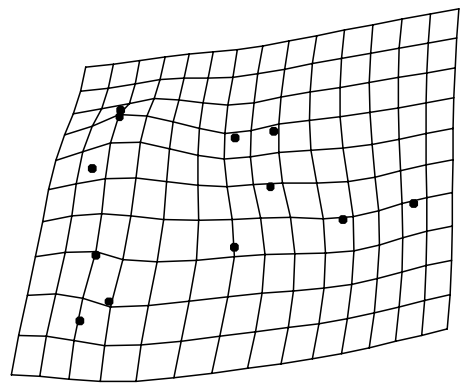

Fig. 14 Analysis of 22 specimens, 12 landmarks as in Fig. 10

interventions on growth, interventions that might be clinical (as in orthodontics) or instead genetic (as in research into craniofacial growth of knockout animal models by Hallgrimsson and others; for an example in this connection where size per se is part of the experimental signal, see Schwarze et al. 2020). In any of these contexts, to demonstrate a centric allometry like that in Fig. 4 b or Fig. 15e is to reify only one single explanatory factor, physical growth (expansion of tissues), rather than any more complex sort of explanation dealing with embryonic structure, biochemical pathways, functional constraint, genomics, or natural selection.

The dramaturgy here wields the centric model to find a single simple-component subset of a data set, instead of taking the data set as a whole and attempting to model it in toto. I will trace one origin of this research theme, the centrality of environmental determinants of animal development within the single taxon, in the next section. The analysis here mentions but does not investigate the obliqueness of the two allometries in Fig. 10d, for instance, that for the growth of the $H$. sapiens specimens (which turns out to be essentially the same as the static allometry between the two species of Homo) versus that for the sexual dimorphism of Pan. As Fig. 15 shows, that taxon need not be limited to one single species; but the explanations the method affords do indeed have to deal with one single factor, namely, growth allometry. In the title of Gould's great 1966 article, "Allometry and size in ontogeny and phylogeny," it appears that there is a much deeper connection between allometry and ontogeny than between allometry and phylogeny, at least when allometry is treated at this level of morphometric detail.

\section{Discussion}

I do not grant any priority as regards landmark-based allometric studies to the most celebrated of the early 20thcentury approaches, even though it is customarily taken to have launched the method of grids exploited in several of our figures. I mean, of course, the method of "Cartesian transformations" originally published in 1917 by D'Arcy Thompson. That is because I believe Thompson's method 
(A) JHE03(26;12),

Procrustes coordinates

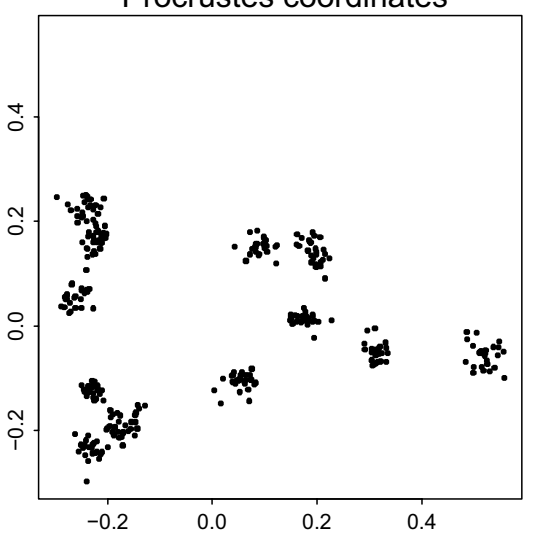

(B) JHE03(26;12),

Proc RW1 vs RW2

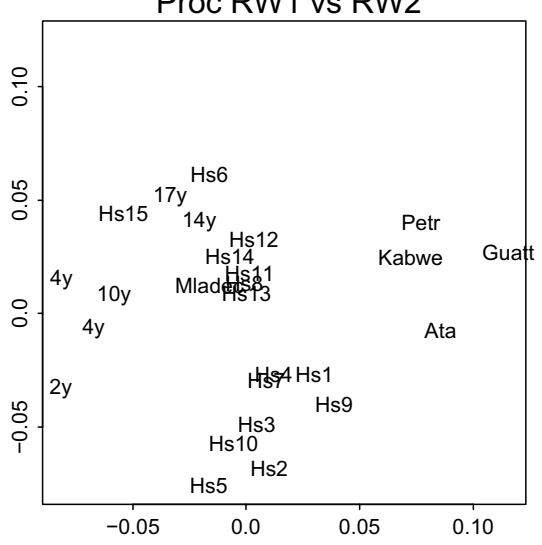

(C) JHE03(26;12),

Boas coordinates

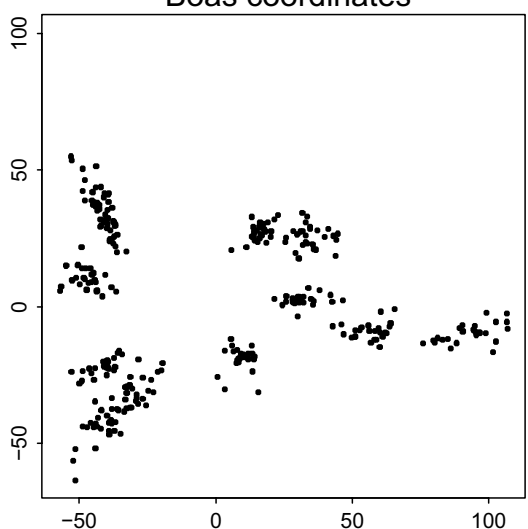

(D) JHE03(26;12),

Boas PC1 vs PC2

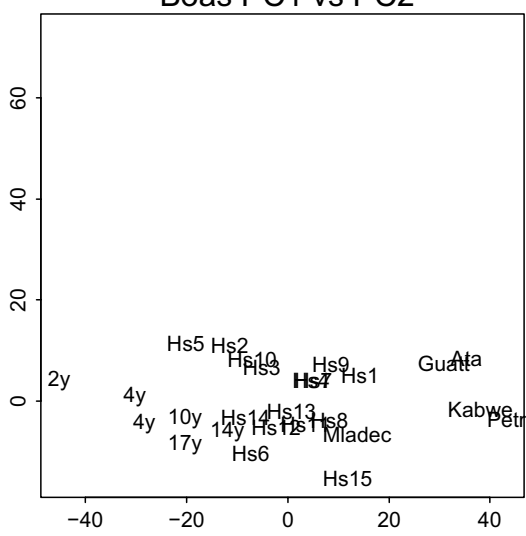

(E) Boas PC1 versus mean form (same scale)

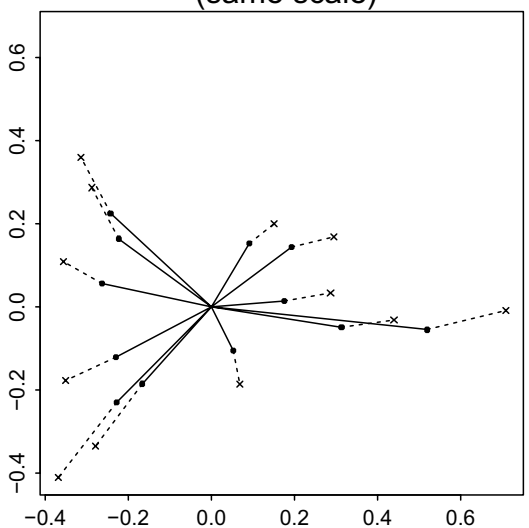

(F) JHE03(26;12), Boas PC1

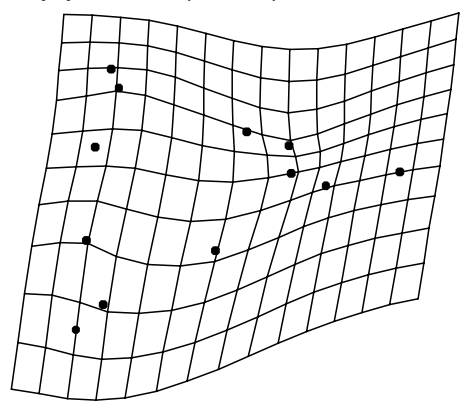

Fig. 15 Concluding analysis of 26 specimens, 12 landmarks as in Fig. 10

to have ultimately proved a dead end rather than a stimulus for the actual development of GMM or any other extant branch of biometrics. (I elaborated on this point in Sect. 5.1 of Bookstein 2018.) In terms of any intellectual history relevant to today's preferred techniques, the next contribution after Boas's of 1905 would be the publications of the Vienna Vivarium, the experimental biology laboratory located at the edge of the great Prater park at some distance from the actual university. Its underappreciated effort to quantify developmental biology was recalled to our attention in the same year as Thompson's centenary via a retrospective symposium and then the proceedings volume edited by its chairman (Müller 2017). The Vivarium not only carried out strikingly original, technically masterful research on the environmental determinants of developmental variation and regeneration (Coen 2006) but also produced many publications in domains we would now classify under the rubric of theoretical biology as that term was interpreted at the time (today's equivalent is probably better taken as "philosophy of biology").

A particularly clarion statement of their programme can be found in Chapter 2, "Organometrie," of Hans Przibram's short pamphlet Aufbau mathematischer Biologie ("The Structure of Mathematical Biology") of 1923. Przibram argues that organismal form must be regarded as the summation of a morphogenesis that is continuous in both space and time, as observed over varying experimental conditions. But because growth is interstitial, we must measure likewise interstitially (that is, inside the organism's boundary) prior to any attempt to summarize via external measurements such as relations among landmarks. The argument continues (in my modern rephrasing) to emphasize that investigations must begin with studies of growth trajectories before they pass on to examine ecophenotypy. And comparisons of species must likewise be considered dynamically, so as to accommodate differences in their developmental conditions, "although these data do not yet afford of a mathematical treatment." In short, organismal form is an epiphenomenon of developmental physiology-a more succinct opposite of the Sokal-Sneath focus on taxonomic concerns cannot be imagined.

For any proponent of a methodology such as Przibram's, Thompson's method of Cartesian transformations cannot be 
JHE03(26;12) Boas nonaffine nonallometric

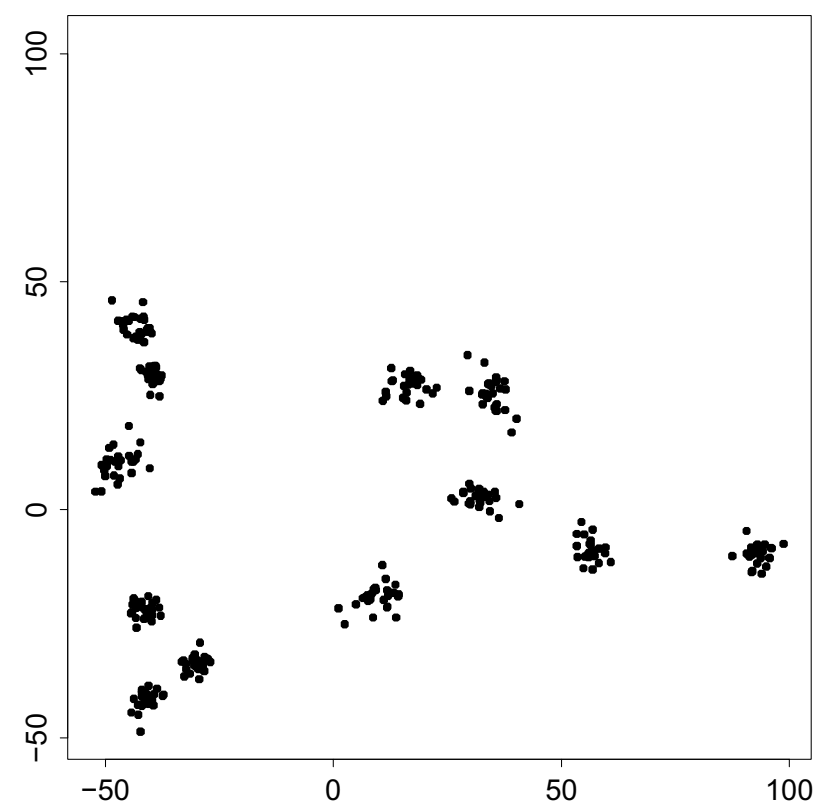

Fig. 16 Residual variation after removing both BPC1 allometry and the affine terms for the 26 12-gons in Fig. 15. The meaning of "nonaffine nonallometric" [shape] is as in Fig. 6

viewed as helpful. In Przibram's critique of 1923, Thompson's method, which he claims (correctly, in my opinion) essentially reduces to comparisons of positions, orientations, and proportions among organs, is purely inductive, and does not conduce to explanations. Indeed, he notes, Thompson gives us no insight into how an organism made up of diverse organs could submit to analysis via a holistic assessment in the first place, let alone change into another species while remaining an integrated, functioning entity. Przibram concludes this frustratingly brief chapter with an insight astonishingly ahead of its time:

Thompson's holistic deformations can be made comprehensible if we can visualize a space lattice upon the living form, so as to assess how each little piece changes its shape under conditions that vary by species. Here lies open a rich, nearly undeveloped field that invites a mathematization, one whose erection we hope will begin very soon. At the conclusion, measurement of the physical space grid will have been unified with stereochemical organometry. (Przibram 1923:14, my translation)

Until the day when observation of gridded tissue all over the developing organism has become a technically feasible mode of primary data collection, Przibram is arguing, Thompson's error is fundamental: those little grid square distortions cannot share any biological reality with the empirically identifiable homologous points at which the data, such as they were, made contact with geometry. Sneath, writing almost half a century later, was careful not to make this error. To him, the grids are for purely descriptive taxonomic purposes, in effect, tools of visual psychology rather than biometry-none of his examples deal with organismal growth. Whereas, Przibram's life work (until it was terminated prematurely in Theresienstadt, in 1944) was the erection of an early version of what is now called "evo-devo" in which those little grid cells were indeed the explicit subject of experimental data collection. In my current view the American anthropologist Franz Boas is more appropriately considered the ultimate progenitor of geometric morphometrics than the British natural philosopher and classicist D'Arcy Thompson; and had Przibram lived into the age of the technology he was imagining, founders' credit might well have been shared with him.

What is meant by the "data" in this context needs to be consistent, at least conceptually, with the Przibram experimental setting: the matching of locations between successive images of the same organism, or the implication of such a match via the theoretically fundamental notation of biological homology. I have discussed this in other publications (e.g. Bookstein 2019) that need not be excerpted here. The concept of matching is baked into the formalism of landmark points, the information modality for which this method of centric allometry (along with other applications of both the Boas and the Procrustes methodologies here) was designed. As I explained long ago, in the earliest of treatises on geometric morphometric and the associated thin-plate splines,

landmarks are quite a bit more than instructions for measurement; they are the places at which one's explanations of biological processes ought to be grounded.

(Bookstein 1991, p. 17)

This single requirement- that correspondences across time or across specimens be interpretable as statements encoding a-priori biological theory-distinguishes most of today's GMM praxis from the more industrialized approaches optimized for processing verbose or complex image data sets, particularly in three or four dimensions, that lack a-priori textbook-referenced annotation. For instance, the deformatica computer package (Durrleman et al. 2014) from the INRIA Aramis project team parametrizes its deformations not by landmarks but by "the most variable parts" of their templates. Such "control points" do not logically substitute for landmarks - their locations do not convey prior biological knowledge, but instead are put forward as an a-posteriori computational substitute for such knowledge. Hence, I would argue, their variability is not necessarily tied to meaningfulness in any developmental or evolutionary context, nor would it conduce to descriptions in terms of growth-gradients or the further exploration of the spatial or 
genomic spectrum of variation in these patterns. The goals of deformatica and similar packages, then, must be considered rather more clinical-medical than biological. They align better with group discrimination or classificationwith the purposes of machine vision, in other words - than with the goal of understanding how normal development works globally via the coordination or compensation of processes at different scales in different regions of the form (cf. Mitteroecker et al. 2020). It is hard to imagine how their (admittedly elegant) discrete differential geometry could be matched to the languages whereby patterns of organismal growth come to make biological sense. ${ }^{7}$

To explain one particular aspect of the Boas maneuver we must turn to the semiotics of actual statistical notation. For landmark data that are close to their "template" (in effect, the sample mean) in both size and shape, the Procrustes maneuver is equivalent to treating the landmark configurations as one single data matrix of cases by coordinates and then projecting out all four of the following composite variables: the mean $x$-coordinate, the mean $y$-coordinate, size (taken as Centroid Size), and orientation. Here size and orientation are treated only via their linearizations, which display an unexpected symmetry when plotted as little vectors of displacement at the means of the landmark coordinates: they fall perpendicularly upon the form. The standard Procrustes approach projects out both of these, thereby preserving a certain formal algebraic symmetry of the resulting residual distributions but at a considerable cost, namely, disqualification of the language of least-squares in centimeters. The Boas standardization preserves that least-squares language at the cost of losing access to this mathematical symmetry. In the presence of size allometry, the linearization that I notated in my " $J$-matrix approach" (Bookstein 2014, 2018 and Appendix A.2 here) will not extend with any confidence over more than a fraction of an order of magnitude as regards geometric scale. But it is difficult to claim statistical validity for GMM studies of broader taxonomic regimes anyway. (In my view any validly linearized "morphometrics of macroevolution" is highly unlikely.)

The trade-off sketched here is thus in practice an empirical issue, not an a-priori one. Does the prior literature on the natural history of the taxon under examination suggest that the dimension of scale is dominant enough in its importance that it must not be manipulated, but instead left for explanation? For topics like growth allometry, the answer

\footnotetext{
7 An underappreciated paper by Rohlf and Sokal (1967) made a similarly ironic point without invoking any geometry at all: the purposes of taxonomy can be achieved satisfactorily by an image analysis that randomly scans line drawings rather than "looking for and scoring characters." Note that for this purpose both of their demonstration data sets, the images of the "Caminalcules," Fig. 1, and the mosquito pupae, Fig. 2, were already in rough Boas registration.
}

apparently should be "Yes." The treatment of size and orientation as somehow equipotent on purely geometrical grounds is unnatural for the class of natural-science applications with which analyses of the evolution of development are concerned. Advanced mathematizations of spaces of very high dimension but very weak theory seem now to making great strides in connection with the emergence of large-scale DNA sequencing resources and other megavariate contexts; but that is not the context of applications at which the present methodology is aimed. Even if images can be followed over megavoxels in individual instances of growth, it is extremely difficult to persuasively match more than a few dozen parameters across individual organisms (cf. Przibram 1922, tables). As the embryologist Paul Weiss (a Vienna-born student of Przibram's who successfully fled the Nazis) noted in 1956, "Identical twins are much more similar than any microscopic sections from corresponding sites you can lay through either of them" (Gerard 1958, p. 140). Stated more formally, an appropriately controlled experiment requires, in particular, appropriate control of the level of detail at which data are captured.

In closing I would highlight a contrast between the purpose of centric allometry analyses like these two exemplars and the more commonly encountered task of "modeling a covariance structure," in this instance the covariances of the shape coordinates in Figs. 2a or 15a. The purpose of the analyses here is the visualization of the hypothesis of growth allometry per se, augmented perhaps by the standardization for uniform change that does not alter descriptions of local features of change (cf. Bookstein 2015). The point is not "goodness of fit" but explanation, and the explanation of growth in Fig. 2, in terms of original Cartesian coordinates and least-squares in millimeters, is far more persuasive than that of Fig. 1, in "Procrustes units." The issue of an overall eigenstructure, as in methods of factor analysis (Bookstein 2017), is oblique to that specific task. Put another way, the manipulations here do not change the significance level of the claim of unidimensionality for views like those in Figs. $4 \mathrm{~b}$ or 15 . One can check for sphericity of residuals from the PC1 model, for instance, and the asymptotic chisquare statistics are virtually identical (and highly significant, too, in view of the curvilinearity obvious in either Figs. 1a or $2 \mathrm{a}$ ).

Hence I am not claiming that there is no further structure to be had in this data set, only that neither of our two exemplary samples ( 18 rodents, or 26 hominids) would be a promising testbed upon which to pursue it. Of the total variance of the Boas coordinates in Fig. 15c, 79\% is explained by the variables controlled in Fig. 16. One of these, the affine term, is a completely a-priori function of the mean, a standardization not for size alone but also for any nonlocal aspect of shape change; while the other, the first principal component of the Boas coordinates, clearly dominates already 
according to Fig. 15d. The residual, 21\%, is not worth trying to explain in a sample so arbitrary and unbalanced as this one. By comparison, in the corresponding summary (Fig. 6) for Vilmann's well-designed experimental data set, the fraction of total Boas variance explained by BPC1 along with those affine terms is $97.6 \%$, leaving only $2.4 \%$ to be attributed to the remaining (uncontrolled) individual variation. The next experiment might well be along Przibram's principles, perhaps investigating how diverse contrasts of experimental conditions affect the growth allometries here. Although these residual fractions of variation- $2.4 \%$ versus $21 \%$ - are enormously different, the algebra of the centric allometry model as fitted is the same. This suggests perhaps a wider domain of application than just the mammalian craniofacial complex: for instance, analysis of the growth of analogous hard-tissue complexes bordering soft-tissue contents under constraint by functional stresses and strains. It might also apply to strongly allometric series of forms observed over phylogeny rather than ontogeny, such as the series of vestibular superpositions recommended by Delattre and Fenart more than half a century ago.

Klingenberg's (2016) review of "concepts of allometry in geometric morphometrics" eschews algebraic notation along with any of the associated equations. In spite of these circumlocutions he concludes that the poles of the fundamental bifurcation with which the present paper is concerned, between the methods that treat size and shape as logically separate properties versus the methods where this distinction is dropped, are "logically compatible and should provide results that are comparable in empirical studies" (p. 132). The two examples in this essay clearly contradict that optimistic generalization. Yes, "logically" the Procrustes approach and the Boas approach exploit the same data base of information (the same original Cartesian coordinate arrays) and are interconvertible by purely algebraic tactics, as demonstrated, for instance, in Fig. 5. Yet our community's papers rely on Procrustes-based morphometrics not for its logic (which I have critiqued strenuously in several earlier venues, e.g. Bookstein 2016, 2018) but for its arithmetic, specifically, the geometry of shape coordinates along with the optimal low-dimensional algebraic summaries of their covariance structures. Although Centroid Size and Boas PC1 correlate 0.999 for the rat growth data (Fig. 6a), the decision to divide by Centroid Size resulted in radically different sets of vectors landmark by landmark across the form (Fig. 4) and substantially different ordinations by their scores (Fig. 3). And as Figs. 10 and 11 show, Boas analyses can make sense of principal component scatters that wholly fail to make allometric sense in a Procrustes context. In both the rat growth example and the hominid static allometry example here, the two styles of analysis diverge to a startling extent in their implications for understanding organismal form and its development. (For more on the logic of growth gradients and their pattern analysis see Bookstein (2019).) Note that neither formula, RW1 or BPC1, is aligned with Centroid Size (see Appendix A.3).

More generally, I would argue, the optimism of Klingenberg's 2016 paper is not supported by any explicit formulas for the conversion of either type of linear multivariate finding from one class of methods to the other. Instead his argument may serve mainly ironically: a reason for intentionally elevating explicit algebraic details of an allometric analysis method to a position of priority over verbal summaries in the actual text of empirical reports. It should not be up to the researcher to "prefer one way of thinking or the other," the way Klingenberg phrases the matter on pp. 132-133. No, the choice is not merely a subjective choice of cognitive style. Rather, it demands a match between the formalisms of an algebraic/stochastic model and the rhetorical structure of whatever morphogenetic theory is ostensibly supported (or refuted) by the analysis at hand. And for analysis of allometry, that must be the match between the formula for size and the explanatory role of size, as demonstrated in this essay. Analyses of growth allometry need to rely on measures of size attuned to the allometric explanation ultimately arrived at, and Centroid Size does not align with any such explanation beyond the obvious formulaic null.

Hence the Procrustes construction for landmark data has unreasonably displaced the boundary between concepts of "size" and concepts of "shape" from where it should have been were these notions to apply cogently in a growth study. As the captions of Figs. 6 and 16 indicate, the term "shape" continues to be useful as part of the exegesis for diagrams that follow a prior sequestration of information appropriately referred to as "size." But the requirement that such a "shape" descriptor be produced by some equation relying on the division symbol $\div$ must be replaced by a far more sophisticated language aligned with the underlying purpose of biological explanation. By enforcing an arithmetical division by Centroid Size prior to any further statistical analysis, today's Procrustes-based geometric morphometrics has drifted far from a productive articulation with the biology, biomechanics, and histology of organismal growth and form. To resuscitate that alignment may mandate reversing that size standardization step in spite of its premature ubiquity as part of the GMM toolkit. Boas coordinates are an elementary alternative to that standard GMM, no more difficult than Procrustes shape coordinates to produce and manipulate and compatible with thin-plate splines and other useful representational tools of the toolkit. The Procrustes method construes "shape" as a set of theoretically incoherent proportions-a set of quotients of centered, rotated Cartesian coordinates by a biologically inappropriate measure of "size." This biotheoretical blunder has hobbled geometric morphometrics for more than 25 years. To circumvent it by a maneuver as simple as foregoing that division would help 
restore the centrality of allometric developmental models to the morphometric analyses that would, in turn, reinvigorate the evolutionary biology of organismal form.

\section{Appendix: Centric Allometry Via Boas Coordinates}

Three interlinked morphometric maneuvers have helped this paper bridge the conventional landmark data structures of Procrustes analysis to the proposed method of centric allometry for morphometric description of organismal growth. The first, even though we end up not relying upon it, is the notion of Centroid Size (CS) as the most symmetrical, atheoretical summary of a landmark configuration's size. Second is the interesting relation of that formula to the angular part (the rotation) of the least-squares fit of one configuration to a template. The third is the justification of the centric allometry model per se for explicit modeling of relative growth in terms of this same least-squares principle, in effect replacing CS with a weighted combination of distances so as to serve optimally for the explanation of growth allometry itself by graded rates of extension of these centric distances, without rotation.

\section{A1: From Interlandmark Distances to Centroid Size}

In the absence of any pertinent biomathematical or biomechanical theory, what is a sensible quantity for summarizing variation of size in a sample of landmark configurations? I restrict attention here to the symmetric formulas that treat all landmarks equivalently, excluding formulas that sort the landmarks into anatomical or functional "modules," for instance, so that the data can only be regarded as landmark locations $l_{p s}$ where $p$ ranges over an otherwise unordered assortment of named points 1 through $k$ and $s$ indexes the specimens of a sample. Let us write $\|a-b\|$ for the ordinary Euclidean distance between any two locations $a$ and $b$. As $a$ and $b$ range over the landmark list, these might occasionally be good (i.e., functionally meaningful) size measures one by one. Yet in the absence of a governing functional perspective there are just too many of them-we need a rule for aggregating all the alternatives into a practical summary. And not every potential rule is equally acceptable to the biologist. For instance, two of the more obvious candidates for a net size measure, perimeter and area, have equally obvious problems whenever they are applied to landmark configurations that are not convex or that have interior points. Recall, too, the main text's example of a contrived scenario where these two options are not consistent in their direction of change.
In our typical organismal context where any interlandmark distance $\left\|l_{p_{1} s}-l_{p_{2} s}\right\|$ might be as interesting a-priori as any other, it would be better to have recourse to one customary default rule for assembling all the alternatives into one summary quantity. This would be the square root of the sum of squares of the whole roster: the formula

$$
\mathrm{CS}_{s}^{\text {allpairs }}=\sqrt{\sum_{\substack{p_{1}=1, \ldots,(k-1) \\ p_{2}=\left(p_{1}+1\right), \ldots, k}}\left\|l_{p_{1} s}-l_{p_{2} s}\right\|^{2}}
$$

applied specimen by specimen. In a happy algebraic coincidence that has been recognized for centuries, this formula is exactly proportional to a simpler one,

$\mathrm{CS}_{s}=\sqrt{\sum_{p=1, \ldots, k}\left\|l_{p s}-C_{s}\right\|^{2}}$,

where $C_{s}$ is the centroid of the landmark configuration for specimen $s$, the average of all $k$ of its separate landmark locations.

This paper exploits this second specific formula, Centroid Size (CS) without the "allpairs" superscript, but not in the way usual for a Procrustes analysis, to wit, as a divisor for "standardizing" size en route to the construction of "shape coordinates." Instead, it will be used to qualify a complementary formula, one geometrically perpendicular to Centroid Size, that provides the least-squares version of a different standardization, the standardization of orientation. As the text has shown, there are alternatives to CS (e.g. the composite BPC1) that can correlate remarkably well (e.g., in the range of 0.999) with CS but that bear coefficients having far more direct interpretations in terms of differential growth, presumably the subject of the allometric studies that are our concern here.

\section{A2: Rotation as the Linearized Complement to Centroid Size for Landmark Data}

Of the four standardizations invoked in the course of producing Procrustes shape coordinates, the Boas procedure will preserve three. It is helpful to examine a formulation, if only an approximate one, according to which all four can be viewed in parallel, so they may be compared and contrasted. This is the formalism of the J-matrix introduced in my textbooks of 2014 and 2018.

The $J$-matrix formalism is a notation for exploring the range of linear combinations of landmark coordinates as they might be used either for scoring or for standardizing. To write it down, we need some notation. Consider a template (likely an average shape) $\mu$ 
on $k$ landmarks $\left(x_{1}, y_{1}\right),\left(x_{2}, y_{2}\right), \ldots,\left(x_{k}, y_{k}\right)$ vectorized as $\left(x_{1}, y_{1}, x_{2}, y_{2}, \ldots, x_{k}, y_{k}\right)$ with $\Sigma x_{i}=\Sigma y_{i}=\Sigma x_{i} y_{i}=0$, $\Sigma\left(x_{i}^{2}+y_{i}^{2}\right)=1$ (meaning: $\mu$ is centered, its Centroid Size is 1 , and it has been rotated to principal axes horizontal and vertical). Write $\delta=1 / \sqrt{k}$. Finally, examine the matrix $J$ of four rows by $2 k$ columns

$J=\left(\begin{array}{ccccccc}\delta & 0 & \delta & 0 & \ldots & \delta & 0 \\ 0 & \delta & 0 & \delta & \ldots & 0 & \delta \\ -y_{1} & x_{1} & -y_{2} & x_{2} & \ldots & -y_{k} & x_{k} \\ x_{1} & y_{1} & x_{2} & y_{2} & \ldots & x_{k} & y_{k}\end{array}\right)$

This matrix $J$ is meant to be applied to deviations from the mean form in the vicinity of that mean, once the appropriate standardizations have been applied to that mean form. (More accurately, the matrix $J$ pertains to deviations in the tangent structure to Procrustes shape space at that mean shape.) The list of those standardizations remains under our explicit control. In the method of centric allometry that is the subject of this paper, we invoke only three of those four standardizations: those corresponding to the first three rows of $J$ but not the fourth.

Note that this prototype $\mu$ has been notated according to its position in Procrustes shape space: centered, at Centroid Size (sum of squares of all its landmarks' Cartesian coordinates) equal to 1.0 , and at a conventional orientation. The average of the Boas coordinates reweights each specimen's contribution to that Procrustes average by multiplying back in the specimen's own Centroid Size, the quantity divided out in the Procrustes procedure. In the presence of a strong allometric component, inasmuch as the Boas average will weight the larger forms somewhat more heavily than the Procrustes average does, the Boas average should express this feature to a slightly greater extent than the Procrustes average. Hence the deformation expressing the one average in terms of the other should strongly resemble the deformation expressing this allometric component, the one that we have already examined in Figs. $1 \mathrm{~b}$ and $2 \mathrm{~b}$ and which Fig. 5 has shown to be well-defined. This expectation is easily verified by a fortyfold extrapolation (Fig. 18) of the corresponding thin-plate spline. (The extrapolation by a factor $\alpha$ of the thin-plate spline from $S$ to $T$ is the thin-plate spline from $S$ to $S+\alpha(T-S)$.) The magnitude of a single-factor shift like this from the scaled Procrustes mean to the Boas mean is proportional to the squared coefficient of variation of Centroid Size; for the Vilmann data here, that upper limit is $2 \%$.

The rows of $J$ are all mutually geometrically orthonormal as $2 k$-vectors (meaning, all four are of unit length and perpendicular in pairs). For the Mardia-Dryden distribution of shapes arising from identical circular Gaussians at every landmark independently (Dryden and Mardia 2016), this means that the scores it supplies are uncorrelated and of the same variance-but that is not our context here: the interest is in those elements of $J$, not the scores they afford on that biologically absurd model. Taking the product of the Boas coordinates with the entries of the first row of $J$ and summing, one arrives at $\delta$ times the sum of the $x$-coordinates of our set of $k$ landmarks, and likewise the second row of $J$ generates $\delta$ times the sum of the $y$-coordinates. Dividing either sum by $k \delta=\sqrt{k}$, then, yields the averages that we should subtract from each $x$-coordinate or $y$-coordinate, respectively, to center the configurations - to put the average landmark of each configuration at exactly $(0,0)$, the origin of Cartesian coordinates. Without further ado, presume this to have been done.

Now examine the lower two rows of $J$. It helps to draw them out as two sets of $k$ little vectors centered on the mean locations of those landmarks (Fig. 17, left). Each pair of vectors makes a little square at each landmark mean position here-for each landmark index $p$, the vectors $\left(x_{p}, y_{p}\right)$ and $\left(-y_{p}, x_{p}\right)$ are the same length and make a $90^{\circ}$ angle - and the size of each square is directly proportional to its landmark's average distance $\sqrt{x_{p}^{2}+y_{p}^{2}}$ from the centroid at $(0,0)$. It is also instructive to trace the new configurations given by the endpoints of either set of vectors (Fig. 17, right).

Together these figures make it visually obvious that the fourth row of $J$, drawn in the solid vectors, encodes change in size only, increase in every distance from the centroid by the same fraction (the same growth rate). To demonstrate this algebraically, note that the differential embodied in the CS formula-the expression of a change in CS as a function of change in any or all of the coordinates of the landmarks - is proportional to the effects of all the infinitesimal changes $d x_{p}$ or $d y_{p}$ of landmark position on the computed CS. Any such change $d x_{p}$, say, induces a change $\frac{k-1}{k} d\left(x_{p}^{2}\right) \sim 2 \frac{k-1}{k} x_{p} d x_{p}$ in the net $\mathrm{CS}^{2}$. (The prefactor $\frac{k-1}{k}$ is there because shifting any single landmark shifts the centroid by $1 / k$ of this shift.) Apart from that prefactor, this is twice the change in $x_{p}$ times its average value. But the odd-numbered coefficients in the fourth row of $J$ are precisely in proportion to these, and, similarly, the evennumbered coefficients that multiply $d y_{p}$ are proportional to $y_{p}$. We are actually using the square root of this sum of squares; this ends up cancelling out the factor of 2 here. (And also there is a factor of the reciprocal of the average CS, but that will not matter, either, as it applies to every specimen in exactly the same way.)

Then the net effect on Centroid Size of moving a landmark from $\left(x_{p}, y_{p}\right)$ to $\left(x_{p}+d x_{p}, y_{p}+d y_{p}\right)$ will be proportional to the projection of the shift $\left(d x_{p}, d y_{p}\right)$, whatever it is, on the vector $\left(x_{p}, y_{p}\right)$. But this is just the vector connecting that mean landmark position to the origin $(0,0)$, which is proportional to the corresponding little solid segment of Figure 17 (left). Assemble them all as a $2 k$-vector and you 


\section{two features of centered shape}

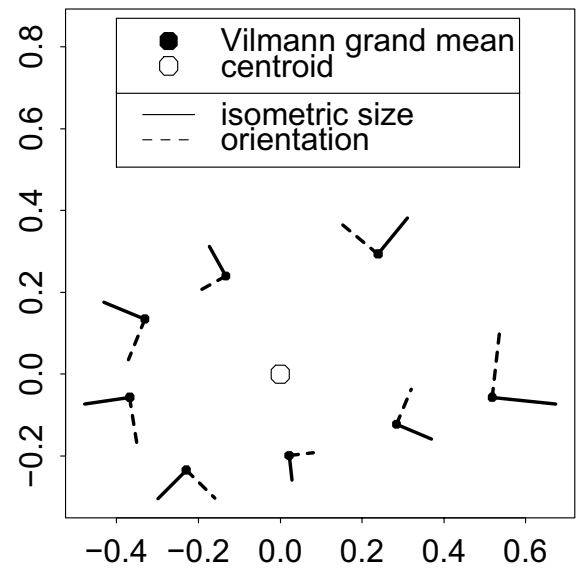

Fig. 17 The geometric relation of orientation standardization and size standardization in the Procrustes method. (left) The linearized contributions to net Euclidean distance of changes in size (solid lines) and changes in orientation (dotted lines) are equal in amplitude and lie at $90^{\circ}$ landmark by landmark, making a little square corner whose scale

\section{From the Proc mean to the Boas mean, $x 40$}

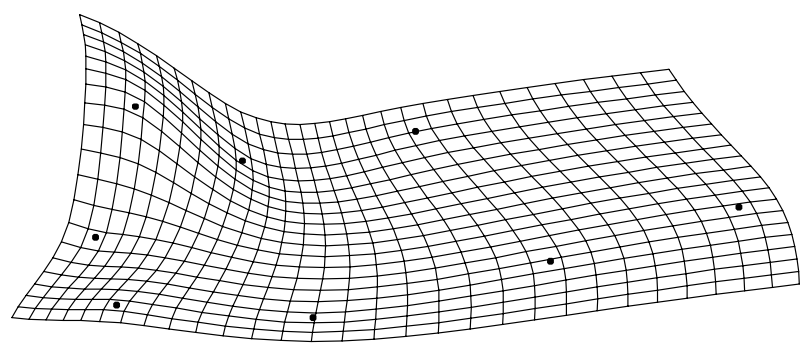

Fig. 18 The deformation from the Procrustes mean shape to the Boas mean form, extrapolated fortyfold. This grid is nearly identical to the grids in Figs. 1b or 2b. See text

get precisely row 4 of the $J$-matrix. In short, then, this row 4 must be encoding a linearization of changes in Centroid Size as all the landmarks move around, any which way, with respect to the template. In the Procrustes method, we can use this insight to get rid of size variation. But in the Boas method here, we carefully protect this same construct from being eroded by any other standardization.

The relevant other standardization is the one encoded in the third row of $J$ and drawn as dashed vectors at left in Fig. 17. From the equivalent rendering in the right-hand panel it is plain that this set of coordinated shifts encodes mainly change in orientation, rotation of all vectors out of the centroid by the same angle (together with a tiny increase in size that vanishes in the limit of small rotations). As noted in the figure legend, while changes in size are "OK" for two types of change

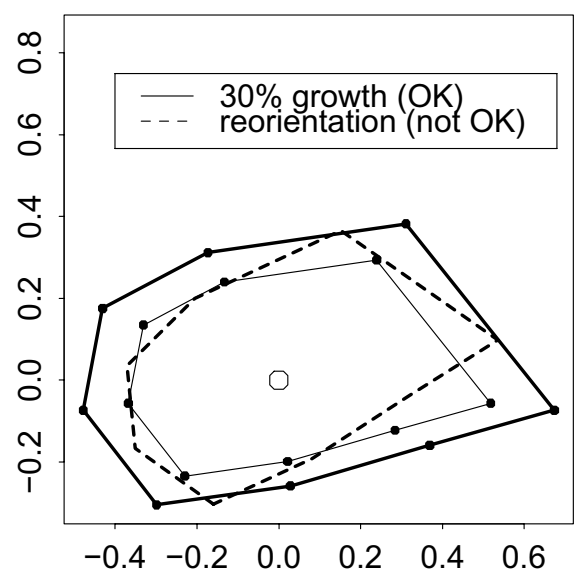

is proportional to the landmark's distance from $(0,0)$. (right) Hence standardizations of orientation and of size by least-squares are geometrically as well as logically independent. The Procrustes method enforces both of these, the method of centric allometry via Boas coordinates, only the orientation requirement

coordinate systems that can be used in studies of growth allometry, changes in orientation are "not OK"; so these will continue to need to be standardized. The amount of rotation applied is exactly proportional to some multiple of the sum of this third-row coefficient vector's elements times the shifts of position between the sample average form and the specimen configuration. In practice, one need not worry about the constant of this proportionality, because it is imposed automatically in the course of the conventional Procrustes registration.

But Boas's article of 1905 already showed that this remaining standardization can be accomplished by a leastsquares procedure, one that is worth rewriting in modern style. Here I borrow from one standard notation for the companion technique of Procrustes fits. When we write out the Procrustes process of fitting one landmark configuration to the translation, rotation, and rescaling of another by least-squares, we find these same four components of $J$ in the standard formulas for the (two-dimensional) regression as written using complex numbers. It will help to write these two configurations as two vectors $\left(z_{1}, z_{2}, \ldots, z_{k}\right)$ and $\left(w_{1}, w_{2}, \ldots, w_{p}\right)$ where the $z$ 's and the $w$ 's are all complex numbers that have already been centered (written here as $\Sigma z_{i}=\Sigma w_{i}=0$ ). Then the superposition we are seeking, the one that minimizes the summed squared deviations of the paired landmarks after the superposition of some scaled and rotated version of the centered configuration $w$ on the centered configuration $z$, will be the solution of the regression problem $z_{i}=\beta w_{i}+$ error, where $\beta$ is some complex coefficient. The textbooks tell you that the least-squares $\beta$ is just the complex number 
$\Sigma z_{i} \overline{w_{i}} / \Sigma w_{i} \overline{w_{i}}$

where the overbar denotes complex conjugation, the reflection of any complex number in the real axis (i.e. the change of sign of its imaginary component).

Examine this formula closely. The denominator is a positive real number, the sum of squared distances of the landmarks of the $w$-configuration from the origin. This thus represents a scaling term, which we have already agreed to ignore. So ignore it, and turn to that numerator $\Sigma z_{i} \overline{w_{i}}$. Written out in terms of coordinates, by the rules of complex arithmetic (Bookstein 2018, pp. 370-371) this is $\Sigma\left(w_{i x} z_{i y}-z_{i x} w_{i y}\right)$. But this is just the dot product of the vectorized configuration for $z$ with the third row, the row for reorientation, of the matrix $J$ corresponding to the template configuration $w$-the row whose little icons in Fig. 17, landmark by landmark, lie at exactly $90^{\circ}$ to the segments out of the origin, without changing their length.

In other words, the least-squares step of the Procrustes complex regression, minimizing the sum of squared landmark-to-landmark discrepancies after both have been superimposed at the same centroid of $(0,0)$, can be separated into two different optimizations, one for scaling and one for rotation, because squared Euclidean distance permits that same division-any pair of axes at $90^{\circ}$ give you the same sum of squared projections. So we can optimize rotation without considering scaling, and vice-versa.

From this identification of the numerator and denominator of the formula for the Procrustes regression with the third and fourth rows of the $J$-matrix, several helpful assertions follow directly:

- the rotation the Boas superposition requires is the same as that produced by the standard Procrustes toolkit;

- it can be interpreted as a multiple of the net distance-weighted rotational displacement of the target configuration with respect to the template, so when the configurations have been registered either to Boas position or to Procrustes position, this net rotation is zero;

- net weighted rotation can be zeroed this way whether or not one also imposes the Procrustes scaling.

We have now fully justified the approach used in Fig. $2 \mathrm{ff}$. of this paper, of producing the Boas superposition, leastsquares among all superpositions that do not change scale, simply by undoing the division step that is part of the conventional Procrustes algorithm.

For a deeper dive into this part of the toolkit, see Bookstein (2014), pp. 417-419, or Bookstein (2018), Sect. 5.4.4. The textbook $J$-matrix has two additional rows for the uniform component of the Procrustes approach, but we will not need these for this paper.

\section{A3: Centric Allometry as a Further Least-Squares Computation}

We are almost done. Consider again the regression coefficient $\beta$ for Procrustes superposition of which both numerator and denominator were just explained, and now apply it to the final step in the Procrustes algorithm, by which every original landmark configuration is superimposed over the Procrustes mean shape by its own least-squares optimization. In the course of this final step, prior to the averaging, each configuration is evidently weighted by the reciprocal of its own Centroid Size $\sqrt{\sum_{p=1, \ldots, k}\left\|l_{p s}-C_{s}\right\|^{2}}$. Indeed such a weighting is required for the standard Procrustes algorithm (Gower's "generalized Procrustes analysis," GPA) to converge to the correct Procrustes average. But for studies of growth allometry, details of that average are of no particular scientific importance; and the cost (the imposition of a weighting that declines in precisely inverse proportion to a specimen's actual size) is severe. If the study is of growth allometry, what could be an adequate justification for imposing such a weighting?-why should the information about the configuration borne by the larger forms be subordinated this way to the information borne by the smaller ones? In any other context, the regression of one variable $x$ against another $y$ when both are monotone-increasing with age would be an ordinary least-squares analysis of one against the other.

Embracing this least-squares theme, then, if it is possible to study growth allometry (dependence of the landmark configuration on size) by a technique that does not require any atheoretical weighting inverse to size, such an approach should be preferred. It is inconvenient that Julian Huxley's style of relative growth modeling, the resort to logtransformed measurements, cannot be used here, inasmuch as shape coordinates do not conduce to a transformation to logarithms. (After the centering step, they are as often negative as positive.) And for any landmark count greater than three, a least-squares analysis of all of the interlandmark distances would have too many parameters, to wit, $k(k-1) / 2$ instead of the $2 k-3$ actually in hand, or else we could simply evoke Jolicoeur and Mosimann's (1960) technique of loglinear principal components.

But recall the least-squares property of principal components: the interwoven facts that (i) the score on the first PC is the linear combination that predicts the full range of variable values with the smallest summed error variance, while at the same time (ii) its coefficients are proportional to the covariances of its scores with those same variables. (See, 
e.g., Bookstein 2018, Sect. 4.2.2.) By exploiting what we already know about principal components, we can combine the least-squares characterization of the Boas coordinates themselves (least-squares in translation and rotation) with the least-squares property of principal component analysis (least-squares in prediction error variance) and thereby arrive at the centric allometry model that has been fitted in the examples of this essay. Although the formula for Centroid Size appears to be installed directly at the root of the Procrustes method, its algebra shows it to be complementary to the orientation term in a way that can be cancelled from the formalism at the end without affecting any other aspect. Once that is done, the superposition (the creation of Boas coordinates) and the growth analysis (the fit of linear explanatory patterns) can proceed in the same least-squares context of predicted growth increments (in units of $\mathrm{cm}$ ) and minimized summed squared unexplained distances (in units of $\mathrm{cm}^{2}$ ), just the dimensions one expects for the components of a biologically cogent growth allometry description.

Clause (ii) in the preceding paragraph is a subtle one, well worth unpacking. It conveys the geometric reason for focusing on BPC1 as the measure of size in the method of centric allometry put forward here: not that it "maximizes the variance" (of the associated explanation) - that is a merely statistical justification-but because the formula for a principal component is what the mathematicians call an eigenvector or characteristic vector - call it $E$ - of a covariance matrix $S$. This means that $E$ is a solution of the characteristic equation $S E=\lambda E$, where $\lambda$ is a decimal number (a constant of proportionality). In our context of centric allometry, the covariance matrix $S$ pertains to the distances from the centroid to the several landmarks of the analysis, as in Figs. $4 \mathrm{~b}$ or $13 \mathrm{c}$, and the eigenvector $E$ is the pattern of proportional extensions of this centric distance landmark by landmark over the course of growth. Then property (ii) above of solutions $E$ of the characteristic equation means that the coefficients of the Boas linear composite BPC1 are strictly proportional to the covariances of the corresponding linear composite score with the landmark-by-landmark distances to the landmarks' common centroid. The formula for BPC1 thereby serves the function that Centroid Size was originally hoped to serve: the composite measure (uniquely) suited to its explanatory role in analyses of allometry. (What disqualifies Centroid Size for this purpose is precisely its actual formula, as graphed in the left panel of Fig. 17. Each landmark's coefficients are proportional to the landmark's mean position with respect to the centroid, not the linearized dependence of its distance from that centroid upon the joint cause of all the simultaneous growth increments.) The contrast between this fundamental characterization of PC's as eigenvectors and their more familiar "justification" as variance-maximizers is well worth keeping in mind. (For more on the algebraic basis of principal components, see any of the standard multivariate textbooks, for instance, Mardia et al. 1979.)

So the centric allometry model here is a landmark-compatible version of an unexpectedly simple fit for a familiar classic model, the single principal component of Jolicoeur and Mosimann (1960) or Blackith and Reyment (1971), second edition, Reyment et al. (1984), as applied to the set of distances (not their logarithms) of the several landmarks out of their common centroid. When, as in Fig. 15e, we see so little apparent additional rotation over growth of the segments joining each landmark to the centroid, the net effect of growth can be described adequately by the growth rates of those lengths out of the centroid - the same lengths that contributed to the definition of Centroid Size in the first place, but now weighted by their growth rates (in centimeters) rather than their mean lengths. Centric allometry in the sense of this paper is thus an interpretation of a landmark covariance pattern as one for which Boas PC1 turns out to be the unique correct definition of size, the optimal substitute for CS: a weighted sum of those same lengths out of the centroid, without any further reference to rotations now that each configuration has already been rotated rigidly according to the score along row 3 of the $J$-matrix. A figure like $4 \mathrm{~b}$ or $15 \mathrm{e}$ is the graphic representation of such a simplified model, a principal component that needs only one parameter, not two, for each landmark of the configuration.

Acknowledgements I have benefitted greatly from continual discussions with Joe Felsenstein, Department of Genome Sciences, University of Washington ever since I moved to Seattle in 2005. (The name "Boas coordinates" was, in fact, Joe's idea.) The paper's initial conceptualization came in the course of conversations at the GMAustria meeting (Department of Botany, University of Vienna, March 2019) with Nicole Grunstra, Sonja Windhager, and especially Philipp Mitteroecker, who served as co-discussant with me throughout that conference. Jim Rohlf (Stony Brook University) sent extensive comments on an earlier draft of the manuscript. The relevance of the Vienna Vivarium's work to the approach here was brought to my attention in conversations with Gerd Müller and Daniel Nicholson of the KLI Klosterneuburg.

\section{Compliance with ethical standards}

Conflict of interest The author has no conflicts of interest in connection with any of the contents of this article, preparation of which was not supported by any external funding source.

Open Access This article is licensed under a Creative Commons Attribution 4.0 International License, which permits use, sharing, adaptation, distribution and reproduction in any medium or format, as long as you give appropriate credit to the original author(s) and the source, provide a link to the Creative Commons licence, and indicate if changes were made. The images or other third party material in this article are included in the article's Creative Commons licence, unless indicated otherwise in a credit line to the material. If material is not included in the article's Creative Commons licence and your intended use is not permitted by statutory regulation or exceeds the permitted use, you will need to obtain permission directly from the copyright holder. To view a copy of this licence, visit http://creativecommons.org/licenses/by/4.0/. 


\section{References}

Blackith, R. E., \& Reyment, R. A. (1971). Multivariate morphometrics. Academic Press.

Boas, F. (1905). The horizontal plane of the skull and the general problem of the comparison of variable forms. Science, 21, 862-863.

Bookstein, F. L. (1981). Comment on "Issues related to the prediction of craniofacial growth.”. American Journal of Orthodontics, 79, 442-448.

Bookstein, F. L. (1983). The geometry of craniofacial growth invariants. American Journal of Orthodontics, 83, 221-234.

Bookstein, F. L. (1986). Size and shape spaces for landmark data in two dimensions. Statistical Science, 1, 181-242.

Bookstein, F. L. (1989). "Size and shape": a comment on semantics. Systematic Zoology, 38, 173-180.

Bookstein, F. L. (1991). Morphometric Tools for Landmark Data: Geometry and Biology. Cambridge: Cambridge University Press.

Bookstein, F. L., Gunz, P., Mitteroecker, P., Prossinger, H., Schaefer, K., \& Seidler, H. (2003). Cranial integration in Homo: singular warps analysis of the midsagittal plane in ontogeny and evolution. Journal of Human Evolution, 44, 167-187.

Bookstein, F. L. (2012). Random walk as a null model for high-dimensional morphometrics of fossil series: geometrical considerations. Paleobiology, 39, 52-74

Bookstein, F. L. (2014). Measuring and Reasoning: Numerical Inference in the Sciences. Cambridge: Cambridge University Press.

Bookstein, F. L. (2015). Integration, disintegration, and self-similarity: characterizing the scales of shape variation in landmark data. Evolutionary Biology, 42, 395-426.

Bookstein, F. L. (2016). The inappropriate symmetries of multivariate statistical analysis in geometric morphometrics. Evolutionary Biology, 43, 277-313.

Bookstein, F. L. (2017). A method of factor analysis for shape coordinates. American Journal of Physical Anthropology, 164, 221-245.

Bookstein, F. L. (2018). A Course in Morphometrics for Biologists. Cambridge: Cambridge University Press.

Bookstein, F. L. (2019). Reflections on a biometrics of organismal form. Biological Theory, 14, 177-211. https://doi.org/10.1007/ s13752-019-00320-y.

Burnaby, T. P. (1966). Growth-invariant discriminant functions and generalized distances. Biometrics, 22, 96-110.

Coen, D. R. (2006). Living precisely in fin-de-siécle Vienna. Journal of the History of Biology, 39, 493-523.

Cole, T. M. III, (1996). Historical Note: Early anthropological contributions to "geometric morphometrics.". American Journal of Physical Anthropology, 101, 291-296.

Corruccini, R. S. (1981). Analytical techniques for Cartesian coordinate data with reference to the relationship between Hylobates and Symphalangus (Hylobatidae: Hominoidea). Systematic Zoology, $30,32-40$.

Delattre, A., \& Fenart, R. (1960). L'hominisation du crâne: étudiée par la méthode vestibulaire. Éditions du CNRS.

Dryden, I. V., \& Mardia, K. V. (1998). Statistical shape analysis. Hoboken, NJ: Wiley.

Dryden, I. V., \& Mardia, K. V. (2016). Statistical shape analysis (2nd ed.). Hoboken, NJ: Wiley.

Durrleman, S., Prastawa, M., Charon, N., Korenberg, J. R., Joshi, S., Gerig, G., et al. (2014). Morphometry of anatomical shape complexes with dense deformations and sparse parameters. NeuroImage, 101, 35-49.

Gerard, R. W., ed. (1958). Concepts of Biology. Publication 560. Washington: National Academy of Sciences.

Gould, S. J. (1966). Allometry and size in ontogeny and phylogeny. Biological Reviews, 41, 587-638.
Gower, J. C. (1975). Generalized Procrustes analysis. Psychometrika, $40,33-51$

Gunz, P. M., Neubauer, S., Maureille, B., \& Hublin, J.-J. (2010). Brain development after birth differs between Neanderthals and modern humans. Current Biology, 20, R921-O922.

Huxley, J. (1932). Problems of Relative Growth. London: Methuen.

Jolicoeur, P., \& Mosimann, J. E. (1960). Size and shape variation in the painted turtle. A principal component analysis. Growth, 24, 339-354.

Klingenberg, C. P. (2016). Size, shape, and form: concepts of allometry in geometric morphometrics. Development, Genes and Evolution, $226,113-127$.

Latour, B. (1990). Drawing things together. In M. Lynch \& S. Woolgar (Eds.), Representation in Scientific Practice (pp. 19-68). Cambridge, MA: MIT Press.

Mardia, K. V., Kent, J. T., \& Bibby, J. (1979). Multivariate Analysis. Hoboken, NJ: Wiley.

Medawar, P. B. (1945). Size, shape, and age. In W. E. LeGros Clark \& P. B. Medawar (Eds.), Essays on Growth and Form presented to D'Arcy Wentworth Thompson. Oxford: The Clarendon Press.

Mitteroecker, P. M., Gunz, P., Bernhard, M., Schäfer, K., \& Bookstein, F. L. (2004). Comparison of cranial ontogenetic trajectories among great apes and humans. Journal of Human Evolution, 46, 679-697.

Mitteroecker, P. M., Bartsch, S., Erkinger, C., Grunstra, N. D. S., Le Maître, A., \& Bookstein, F. L. (2020). Morphometric variation at different spatial scales: coordination and compensation in the emergence of organismal form. Systematic Zoology, 69, 913-926.

Moss, M. L., Skalak, R., Shinozuka, M., Patel, H., Moss-Salentijn, L., Vilmann, H., et al. (1983). Statistical testing of an allometric centered model of craniofacial growth. American Journal of Orthodontics, 83, 5-18.

Moss, M. L., Skalak, R., Patel, H., Shinozuka, M., Moss-Salentijn, L., \& Vilmann, H. (1984). An allometric network model of craniofacial growth. American Journal of Orthodontics, 85, 316-332.

Moyers, R. E., \& Bookstein, F. L. (1979). The inappropriateness of conventional cephalometrics. American Journal of Orthodontics, 75, 599-618.

Müller, G. B. (Ed.). (2017). Vivarium: Experimental, Quantitative, and Theoretical Biology at Vienna's Biologische Versuchsanstalt. Cambridge, MA: MIT Press.

Neubauer, S., Hublin, J.-J., \& Gunz, P. (2018). The evolution of modern human brain shape. Science Advances, 4(1), eaao5961.

Phelps, E. M. (1932). A critique of the principle of the horizontal plane of the skull. American Journal of Physical Anthropology, $17,71-108$

Przibram, H. (1922). Form und Formel im Tierreiche. Beiträge zu einer quantitativen Biologie, I-XX. Franz Deuticke.

Przibram, H. (1923). Aufbau mathematischer Biologie. Abhandlungen zur theoretischen Biologie, vol 18. Gebrüder Bornträger.

Reyment, R. A., Blackith, R. E., \& Campbell, N. A. (1984). Multivariate morphometrics (2nd ed.). Academic Press.

Reyment, R. A., \& Jöreskog, K. H. (1993). Applied Factor Analysis in the Natural Sciences. Cambridge: Cambridge University Press.

Rohlf, F. J., \& Slice, D. E. (1990). Methods for comparison of sets of landmarks. Systematic Zoology, 39, 40-59.

Rohlf, F. J., \& Sokal, R. R. (1967). Taxonomic structure from randomly and systematically scanned biological images. Systematic Zoology, 16, 246-260.

Schwarze, U. Y., Dobsak, T., Gruber, R., \& Bookstein, F. L. (2020). Anatomical similarity between the Sost-knockout mouse and sclerosteosis in humans. Anatomical Record, 303, 2295-2308. plus cover.

Sneath, P. H. A. (1967). Trend-surface analysis of transformation grids. Journal of Zoology, 151, 65-122. 
Sokal, R. R., \& Sneath, P. H. A. (1963). Principles of Numerical Taxonomy. New York: W H Freeman Co.

Thompson, D'. A. W. (1917). On Growth and Form. Cambridge: Cambridge University Press.

Todd, J. T., \& Mark, L. S. (1981a). Issues related to the prediction of craniofacial growth. American Journal of Orthodontics, 79, 63-80.
Todd, J. T., \& Mark, L. S. (1981b). A reply to Dr. Bookstein. American Journal of Orthodontics, 79, 449-455. 